\title{
Reform und Geschichte
}

\section{Das Beispiel der ersten Großen Koalition 1966-1969}

\section{Kurzfassung}

Politik bedarf der Begründung und immer wieder auch der expressiv-symbolischen Beglaubigung: von der politischen Idee bis hin zur parlamentarischen Debatte und Entscheidung. Der instrumentell-argumentative Rückgriff auf Geschichte ist dabei so notorisch wie die Verknüpfung mit leitenden Zukunftsbildern. Reformregierungen und -projekte sind in dieser Hinsicht besonders aufschlussreich, da sie in der Regel unter erhöhtem Rechtfertigungsdruck stehen. Die Regierung Kiesinger/Brandt amtierte in einer Zeit vielfältiger politischer und gesellschaftlicher Umbrüche. Die Große Koalition, die sich selbst als „Markstein in der Geschichte“ begriff, setzte diverse größere Reformen auf die politische Agenda, von denen die Mehrzahl auch realisiert wurde. Dies war ohne Politik mit der Geschichte kaum möglich.

Die explorative Studie untersucht an ausgewählten Texten den Zusammenhang von Geschichts- und Reformpolitik und zeigt auf, welche politische Bedeutung und Funktion der historischen Argumentation im Kontext der übergreifenden Begründung dieser Reformen zukam und in welchem Verhältnis dies zur zeitgenössischen westdeutschen Gesellschaft stand. Dabei zeigt sich, dass Geschichtspolitik in Umbruchzeiten prekärer Natur ist: Wo Grundlagen von Politik sich verändern oder völlig neu definiert werden, sind historische Argumentationen oftmals kontraproduktiv zu aktuellen Intentionen. 


\section{Inhalt}

1. Reformpolitik und Geschichtspolitik 292

2. Die Kiesinger-Brandt-Regierung im politischen Kontext 295

3. Fallbeispiele zur Geschichtspolitik der Großen Koalition 301

a) „Markstein in der Geschichte“: Kiesingers Regierungserklärung 302

b) „Heute lässt uns die Geschichte als Lehrmeisterin im Stich“. Die

Ost- und Deutschlandpolitik am Beispiel des Umgangs mit dem Gedenktag 17. Juni

c) „Kein Ermächtigungsgesetz“: Die Notstandsgesetze 311

d) „Bonn ist noch nicht Weimar“: Die Wahlrechtsreform 315

4. Geschichte als Problem - ein Fazit 317

\section{Reformpolitik und Geschichtspolitik}

Kurt Georg Kiesinger, Kanzler der ersten Großen Koalition auf Bundesebene, meinte in seiner Regierungserklärung am 13. Dezember 1966, der alte Satz von der Geschichte als Lehrmeisterin des Lebens helfe nicht mehr weiter, denn die weltpolitische Situation sei einzigartig (Kiesinger 1967: 543). Aus dieser Bemerkung könnte man schließen, die damalige Bundesregierung habe mit Geschichte keine Politik gemacht. Doch auch die Rhetorik, die Geschichte als Orientierungsinstanz auf Distanz zum politischen Handeln bringen möchte, kann dem Modus der Geschichtspolitik nicht entkommen.

Wenngleich die Große Koalition der Jahre 1966 bis 1969 verstärkt Gegenstand der jüngeren Forschung geworden ist, ist sie bislang doch unter dem Aspekt des Umgangs mit Geschichte nur selten näher untersucht worden. Vor diesem Hintergrund bilden im Folgenden zwei allgemeine Komplexe die leitende Fragerichtung in der Analyse der Großen Koalition. Zum einen die in der politikwissenschaftlich-zeitgeschichtlichen Forschung der letzten eineinhalb Jahrzehnte in unterschiedlichen Aspekten beleuchtete Frage, ob Politik Geschichte braucht und welcher Nutzen damit für die betreffenden Akteure verbunden ist (Schmid 2009). Die zweite Frage betrifft hingegen mögliche Relativierungen einer vorschnellen Bejahung der Frage, ob Politik Geschichte braucht, denn ein naheliegender Einwand lautet: Politik braucht Geschichte nur in Abhängigkeit von den sie bestimmenden zeitgenössischen Rahmenbedingungen, es gibt gewissermaßen Konjunkturen für Geschichte in der Politik. $\mathrm{Ob}$ dies auch für die Zeit der Regierung Kiesinger/Brandt zutrifft, ob und wie 
der historische „Griff nach der Deutungsmacht“ (Winkler 2004a) praktiziert wurde, ist hier näher zu prüfen.

Die Rede von Geschichtspolitik meint in einer ersten Annäherung zunächst den vielgestaltigen Umgang politischer Akteure mit dem Konstruktionselement „Geschichte“. ${ }^{1}$ Aus der nicht mehr zu beeinflussenden Sphäre der Vergangenheit werden einzelne Bestandteile zu einer zweckgerichteten politischen Erzählung „montiert“, zu einer selektiven, interessengeleiteten und gegenwartsdominierten Geschichte, einer Teilgeschichte aus dem unendlichen Kosmos der Vergangenheit: Blickt man genauer auf das Untersuchungsfeld, dann zeigt sich, wie die betreffenden Akteure - sei es sprachlich, materiell darstellerisch oder symbolpolitisch - mit Vergleichen, Analogien, Gleichsetzungen oder Unterscheidungen gleichsam historische ,Blaupausen' in legitimierender oder delegitimierender Absicht in Relation zur Gegenwart setzen. Diese Legitimationsfunktion steht im Mittelpunkt geschichtspolitischer Analysen, erschöpft sie jedoch nicht. Verglichen mit anderen politischen Handlungsfeldern ist Geschichtspolitik tendenziell mehr an Bewusstseinsbildung als an Problemlösung orientiert, zielt eher auf mittelbare denn direkte Wirkung. Als sinnstiftende Repräsentation der Vergangenheit soll sie die historisch-politische Identität der jeweiligen Bezugsgruppe prägen und so - konkret oder mittelbar - eine Kontinuität, womöglich gar eine Tradition stützen oder schaffen und letztlich zu einer Handlungsformierung beitragen. Mit Geschichte wird also aktueller, für weitere Operationen anschlussfähiger politischer Sinn generiert. Um es mit Martin Walsers Sarkasmus auszudrücken: „Die Toten werden noch gebraucht" (Walser 1986: 84).

Ein aufschlussreicher Teilbereich dieser politikwissenschaftlich-zeithistorischen Forschungsperspektive ist die Frage nach dem Stellenwert von Geschichtspolitik in ausgewählten Politikbereichen. Sei es Kultur- oder Sozialpolitik, sei es Verteidigungs- oder Wirtschaftspolitik - der Rückgriff auf Geschichte zur Begründung, Durchsetzung und Darstellung von Politik findet in jedem Politikfeld statt, und zwar in zwei Ausprägungen: als instrumentellproblemlösendes sowie als symbolisch-expressives Handeln. Stets geht es dabei um den Einfluss auf die öffentlich wirksamen respektive vorherrschenden Geschichtsbilder und damit um die Prägung kollektiver Gedächtnisse (König 2008). Fragt man nun zunächst übergreifend in typologischer Absicht nach

1 Zur Konzeptdiskussion sowie Abgrenzung zu verwandten Begriffen wie Erinnerungsund Vergangenheitspolitik siehe Schmid 2001: 40-61; Schmid 2008; Schmid 2009; vgl. auch Wolfrum 1999: 25-32; Steinbach 2001; Fröhlich/Heinrich 2004; Kohlstruck 2004. 
dem Modus von Politik, dann stellt sich auch die Frage nach der politischen Funktion von Geschichtspolitik grundsätzlicher. Unter „Modus von Politik“ verstehe ich die übergreifenden Leit-, Verlaufs- und Zielvorstellungen der Akteure. Am bekanntesten sind wohl die Formen „Revolution“ und „Reform“, zu nennen wären auch das dezidierte Bewahren der Verhältnisse (Konservieren) sowie die Rückkehr zu früheren Zuständen (Reaktion) als ebenso charakteristisch moderne Orientierungsmuster des Politischen. Mit derlei Leitimperativen und -narrativen greifen die Akteure und Rezipienten von Politik zur Begründung ihres Handelns und ihrer Zukunftsentwürfe auch auf Geschichtsbilder zurück. Denn die Motivation zu Revolution oder Reform, zum Bewahren oder zur Reaktion - oder zu deren jeweiliger Kritik - liegt auch in deutender Konstruktion von Vergangenheit zu spezifischen Geschichtsbildern ebenso wie im Entwurf leitender Zukunftsvorstellungen. Deshalb bildet die Geschichtspolitik eine zentrale Arena der ",politischen Deutungskultur“ (Rohe 1996: 8 ff.; Rohe 1994: 168 ff.). Nach der Bedeutung von Geschichte für eine spezielle Reformpolitik zu fragen, bedeutet also, sich auf eine Seite der temporalen Politikbegründung und -darstellung zu konzentrieren.

Es geht somit darum, Geschichtsrekurse als Begründungselemente für Reformpolitik zu untersuchen. Grundsätzlich sind dabei zwei Formen zu differenzieren: zum einen ,pejorative' oder ,negative Geschichtsbilder', die eine ungewollte politische Entwicklung abwehren sollen, zum anderen ,affirmative“ oder ,positive Geschichtsbilder', mit denen eine bejahte politische Richtung gefördert werden soll. Dies wird in der folgenden explorativen Studie exemplarisch anhand der Politik der ersten Großen Koalition untersucht. Nach einer Skizze des zeitgeschichtlichen Kontextes der Koalition der Unionsparteien mit der Sozialdemokratie wird der Umgang mit Geschichte in vier Fallstudien näher betrachtet: zunächst die Regierungserklärung Kiesingers, anschließend drei zentrale Politikfelder der Regierung. Die Auswahl der Quellen folgte der Überlegung, zwei Handlungsformen der Regierungsmitglieder geschichtspolitisch zu analysieren: einerseits die symbolpolitisch bedeutsame politische Rede (Kap. 3a und b), andererseits das diskursive Agieren von Ministern im Kontext öffentlicher Kritik an Reformprojekten der Koalition (Kap. 3c und d). Präsentiert werden Textpassagen, die explizit historisch argumentieren respektive Geschichtsbilder enthalten. Im Mittelpunkt der Analyse steht dabei die Frage, ob und inwiefern die großkoalitionären Akteure ,Geschichte` zur Begründung, Darstellung und Durchsetzung der reformpolitischen Agenda nutzten. 


\section{Die Kiesinger-Brandt-Regierung im politischen Kontext}

Die zeitgeschichtliche und politikwissenschaftliche Forschung hat sich bis vor wenigen Jahren nur am Rande mit der Großen Koalition befasst. Was ein Publizist im Jahre 2007 über das zweite Regierungsbündnis aus Unionsparteien und Sozialdemokratie im Bund schrieb, schien lange auch für das erste zu gelten - sie wirkte „wie eine Pause in der Geschichte“ (Schmid, T. 2007). Nur selten wird das Thema „Große Koalition“ im historischen Längsschnitt wahrgenommen (März 2007). Auch schien es zwischen Adenauer-Ära und sozialliberalem „Machtwechsel“ wenig interessante politische Entwicklungen und Entscheidungen zu geben. So konnte ein 1991 erstmals veröffentlichtes Buch nicht ganz zu Unrecht den Titel tragen „Die vergessene Regierung“ (Schmoeckel/Kaiser 2005). Dies hat sich inzwischen erkennbar geändert, nicht zuletzt aufgrund der verbesserten Quellenlage ${ }^{2}$ neuerer Darstellungen, ${ }^{3}$ aber auch infolge des Interesses am Regierungsmodell „Große Koalition“ im Kontext der Merkel-Steinmeier-Regierung der Jahre 2005 bis 2009. ${ }^{4}$ So wird nun deutlicher, dass die knapp drei Jahre amtierende Regierung Kiesinger-Brandt jedenfalls in der Bilanz mehr als eine bloße Notlösung in einer schwierigen parteipolitischen und gesellschaftlichen Situation markierte. Freilich steht die inzwischen herausgearbeitete Faktenlage konträr zu jener bemerkenswerten Leerstelle im öffentlichen Bewusstsein, das nach wie vor keine konkrete Leistung mit dieser Regierung verbindet. ${ }^{5}$

Die Große Koalition - nach Peter A. Halls Typologie eine Reformregierung zweiter Ordnung, die bisherige Zielsetzungen mit neuen Politikinstrumenten verfolgte (Hall 1993: 279) - verabschiedete weit über 400 Gesetze. Darunter waren nicht wenige, die bedeutsame Strukturveränderungen einleiteten: So wurden etwa das BAföG erfunden; die Lohnfortzahlung im Krankheitsfall auch für Arbeiter eingeführt (und damit eine Gleichstellung mit Angestellten erreicht); das Strafrecht modernisiert; die festgefahrene Ost- und Deutschlandpolitik mit ersten freieren Gehversuchen aufgelockert; in der Wirtschaftspolitik die neuen Instrumente der „Globalsteuerung“ und der „konzertierten Aktion“ kreiert; zudem eine tiefgreifende Neuordnung der Finanzverfassung zwischen Bund und Ländern realisiert; und (nach schwierigen Verhandlungen) mit der

2 Vgl. Buchstab 2005; Fabian/Rössel 2009; Tüffers 2009; Marx 2010.

3 Vgl. Kroegel 1997; Schneider 1999; Taschler 2001; Schönhoven 2004; Türk 2006; Rosin 2007; Eichhorn 2009; Strohmeier 2009.

4 Vgl. Schildt 2005; Dittberner 2006; Dittberner 2007; Niclauß 2008.

5 Vgl. Schönhoven 2004; Metzler 2005; Hildebrand 2006. 
DKP auch wieder eine kommunistische Partei zugelassen. Wenngleich die Wirkungen der einzelnen, teils innovativen Reformen oft zwiespältig, aber weitreichend waren und so einer kritischen Würdigung bedürfen, fällt das zeitgeschichtliche Gesamturteil über diese erste Große Koalition auf Bundesebene mittlerweile ausgesprochen positiv aus: Nicht nur ihre ,unbestrittenen gesetzgeberischen Leistungen und außenpolitischen Erfolge" (Winkler 2004b: 267; vgl. auch Morsey 2000: 98-112) werden hervorgehoben. Die Regierung Kiesinger/Brandt habe ,gerade im Bereich der inneren Reformen viele Weichen [...] gestellt“, deshalb könne sie ,insgesamt als eine Art Inkubationszeit für eine demokratische Umgründung der Bundesrepublik betrachtet werden“ (Wolfrum 2005: 299).

Der Umstand, dass es Ende des Jahres 1966 überhaupt zur Bildung einer Koalition von Unionsparteien und Sozialdemokratie kam, war hinsichtlich der auslösenden Faktoren ein Ergebnis zweier Entwicklungen: des Rücktritts sämtlicher FDP-Minister Ende Oktober 1966 sowie des weitreichenden Autoritätsverfalls von Bundeskanzler Ludwig Erhard. Obgleich er die Bundestagswahlen am 19. September 1965 als fulminanter Wahlsieger absolviert hatte, sank sein politischer Stern anschließend rapide. Außenpolitisch geriet die Bundesrepublik unter Erhard erstmals seit ihrer Gründung in eine gewisse Isolierung zu den wichtigsten westlichen Partnern, den USA und Frankreich, wirtschaftspolitisch näherte sie sich zusehends einer Rezession. Letzteres vor allem entzog dem als „Vater des Wirtschaftswunders“ apostrophierten Kanzler den entscheidenden symbolpolitischen Kredit. Im Herbst 1966, nach der Rückkehr von einem nur als herbe politische Niederlage beschreibbaren USA-Besuch, rebellierte die CDU-Fraktion im Bundestag. Erhards umfassender Autoritätsverlust weckte Ängste vor einem baldigen Machtverlust.

Infolge der Demission der liberalen Kabinettsmitglieder, die eine Steuererhöhung zum Haushaltsausgleich ablehnten, stand die CDU vor einem Scherbenhaufen und binnen weniger Wochen vor der Alternative, entweder erstmals im Bund in die Opposition zu gehen oder die Möglichkeit einer großen Koalition auszuloten. Vorzeitige Neuwahlen schloss man gänzlich aus, dazu schien die Ausgangslage der Union zu ungünstig. Zudem weigerte sich der amtierende Kanzler Erhard trotz Verlusts der Regierungsmehrheit zurückzutreten. Daraufhin setzte sich der noch amtierende baden-württembergische Ministerpräsident Kurt Georg Kiesinger am 10. November in einer Kampfabstimmung als neuer Kanzlerkandidat durch. Aufgrund der Zerrüttung des Verhältnisses zur FDP (die FDP sei ,einfach nicht mehr koalitionswillig und koalitionsfähig“, so Kiesinger am 29.11.1966, zit. nach Buchstab 2005: 382) und der dramatisierenden 
politischen Diagnose der CDU-Führung wurden die Weichen früh auf eine Kooperation mit der Sozialdemokratie gestellt, obwohl man sich intern einig war, dass dies ein zwar großes, aber keineswegs erfreuliches Experiment sei (Buchstab 2005: 404). Somit trafen sich partei- und staatspolitische Interessen der Union mit dem defensiven Kalkül der SPD, die die potenzielle Einstimmenmehrheit mit der FDP als zu riskant und ein Bündnis mit den Unionsparteien als ersten Schritt aus der Opposition in die Regierung betrachtete - und diesen als staatspolitische Voraussetzung einer später sozialdemokratisch geführten Regierung einschätzte, konnte doch damit endlich auch im Bund Regierungstauglichkeit demonstriert werden (Schönhoven 2004: 35 ff.).

Diese Krise innerhalb der Union war der Auslöser des Regierungswechsels; die Ursachen reichten freilich tiefer. Neben dem schon erwähnten kritischen Verhältnis zur FDP waren vor allem wirtschafts- und außenpolitische Gründe maßgeblich. In einer Titelgeschichte fragte Der Spiegel schon am 3. Januar 1966 besorgt: „Ist das Wirtschaftswunder zu Ende?“ Die Bundesrepublik sah sich nach 15 Jahren eines beispiellosen ökonomischen Aufstiegs mit einer als ernsthafte ökonomische Bedrohung wahrgenommenen Entwicklung konfrontiert: 1965 war das Bruttosozialprodukt (BSP) noch um 5,7 Prozent gestiegen, 1996 halbierte sich der Zuwachs auf 2,8 Prozent und 1967 fiel das BSP erstmals seit 1949 um 0,2 Prozent. Ende 1966 ging die schleichende Stagnation in eine offene Rezession über. 1967 stieg die Zahl der arbeitslos Gemeldeten schubartig auf über 600.000 an, zudem tat sich ein Haushaltsdefizit von drei Milliarden DM auf. In der Verschärfung dieser in Deutschland aus historischer Erfahrung hochsymbolischen volkswirtschaftlichen Kennziffern (Longerich 1995: 303 ff.; Ullrich 2009: 336 ff.) wurzelte ein großer Teil der öffentlichen Krisenstimmung, mancherorts wurden Analogien zur Wirtschaftskrise von 1929/30 gezogen. Dass der so häufig als „Vater des Wirtschaftswunders“ gepriesene Ludwig Erhard gegen diese Tendenzen machtlos schien, war Krisensymbol genug. „Am Anfang war die Krise“, lautete denn auch Helmut Schmidts Diktum zur Rechtfertigung der Großen Koalition aus dem Jahre 1968 (Schmidt 1968). Überdies geriet die Bundesrepublik Mitte der sechziger Jahre in die akute Gefahr einer außenpolitischen Isolierung, wobei besonders die ost- und deutschlandpolitische Stagnation unter dem späten Adenauer und während Erhards kurzer Regierungszeit zu nennen ist, aber auch die sich zunehmend schwieriger entwickelnden Beziehungen zu Frankreich und den USA.

Neben diesen allgemeinen krisenhaften Veränderungen sprachen jedoch auch diverse Problemkonstellationen in zentralen Feldern der westdeutschen Politik für ein Zusammengehen der beiden Volksparteien. Hier seien nur die 
Wichtigsten genannt: die immer drängendere Bildungs- und Hochschulreform, um der nicht nur von Georg Picht diagnostizierten „Bildungskatastrophe“ (Picht 1964) zu begegnen; ferner die Justizreform, die sich vor allem auf das politische und das allgemeine Strafrecht richtete; hinzu kam die seit fast einem Jahrzehnt, nun aber zunehmend kontrovers diskutierte Notstandsgesetzgebung, die der Großen Koalition den schärfsten politischen Konflikt eintrug. Zudem war unter den beiden großen Parteien und in Teilen der Öffentlichkeit das Bewusstsein gewachsen, das politische System bedürfe einer Korrektur des Wahlrechts, um klare politische Mehrheiten nach dem Vorbild des britischen Zweiparteiensystems zu etablieren. Dies war natürlich gegen die FDP und generell die kleineren, die Rolle des „Züngleins an der Waage“ einnehmenden Parteien gerichtet. Parallel zu den wirtschafts- und außenpolitischen Entwicklungen, die für eine Zusammenarbeit der beiden großen Parteien sprachen, hatte sich ein zusätzlicher innenpolitischer Reformstau gebildet, der die Bundesrepublik plötzlich als Land mit beträchtlichem Entwicklungsbedarf erscheinen ließ. ${ }^{6}$ So gab es für die Handelnden gute Gründe für den Versuch, mit dem jeweils vormaligen Gegner eine Koalition zu wagen. Auch der im November 1966 während der Koalitionsgespräche beginnende Einzug der erst zwei Jahre zuvor gegründeten rechtsextremen Sammlungspartei der NPD in mehrere Landtage wurde als Bestätigung für ein Bündnis der beiden großen Parteien verstanden, während umgekehrt Kritiker der neuen Koalition die Wahlerfolge am rechten Rand gerade als eine Folge der Kiesinger-Brandt-Regierung deuteten (Prasse 2010; Schmid 2007a).

Gleichwohl, der Entschluss zur erstmaligen Bildung einer Großen Koalition auf Bundesebene entfachte in der westdeutschen Gesellschaft eine Welle der Besorgnis, ja teilweise des Entsetzens (Schroers 1968; Knorr 1974). Es „schien alles ganz anders als sonst - ungewisser, problematischer, irgendwie riskanter", stand doch für manche kritische Beobachter ,die Existenz der zweiten deutschen Republik schlechthin" auf dem Spiel (Hildebrand 2006: 611). Insbesondere von liberaler und linksliberaler Seite wurde das Bündnis der SPD mit der CDU/CSU scharf kritisiert. Im Mittelpunkt stand dabei die Minimierung parlamentarischer Opposition und damit der Kontrolle der Regierung: 447 Abgeordnete von CDU/CSU und SPD standen 49 FDP-Parlamentariern gegenüber. Ralf Dahrendorf sprach in diesem Zusammenhang vom „Ende des parlamentarischen Regierungssystems“ (zit. nach Schneider 1999: 225), andere erkannten ,ein Regierungs-Kartell der beiden großen Parteien, das von sich 
aus die Erinnerung an die Gefahren der Weimarer Republik heraufbeschwört" (Schuster 1967: 812). Das die Bundesrepublik seit ihrer Gründung begleitende Weimar-Narrativ, mit dem das Scheitern der ersten deutschen Demokratie in politischen Diskussionen immer wieder - sei es als erneut drohende Gefahr, sei es zur Verdeutlichung der Differenz „Bonn ist nicht Weimar“ (Allemann 1956; Ullrich 2009) - aktualisiert wurde, war in diesen Dramatisierungen oft präsent. Die Szenarien weckten Ängste vor einer autoritären Gesellschaft, verstärkt durch einzelne Projekte der neuen Regierung, vor allem der Notstandsgesetze und der Wahlrechtsreform.

Dass die Sozialdemokratie ein Regierungsbündnis mit jenen politischen Kräften einging, von denen sie vor allem unter Adenauer verunglimpft und gedemütigt worden war, konnte sie nicht nur innerparteilich schwierig vermitteln. Zusätzlichen Schub erhielt diese Kritik durch die Kooperation der SPD namentlich Willy Brandts als Symbol des ,,anderen Deutschland“ - besonders mit zwei Unionspolitikern, die aufgrund ihrer Vergangenheit für einen Teil des linksliberalen Lagers politisch indiskutabel waren. Dabei ging es besonders um den nicht nur von Günter Grass (1969: 100) als ehemaliger „Mitläufer“ bezeichneten Kiesinger, einen der „Märzgefallenen“ von 1933, der bis 1945 NSDAP-Mitglied geblieben war und als stellvertretender Leiter der Rundfunkpolitischen Abteilung des Auswärtigen Amtes fungiert hatte, dabei aber - so die Deutung seines Biografen - etwa seit 1934 als loyaler, jedoch ,stiller Gegner des Nationalsozialismus“ gelten kann (Gassert 2006: 747; Klöckler 2005). Im November 1968 erhielt Kiesinger dafür die wohl berühmteste Ohrfeige der bundesrepublikanischen Politikgeschichte, als Beate Klarsfeld, deren Schwiegervater in Auschwitz vergast worden war, den „obskuren Kanzler" (Böll 1969: 7) auf dem CDU-Bundesparteitag mit dem Ausruf „Nazi!“ touchierte. Die zweite politische Unperson war der durch die „Spiegel-Affäre“ von 1962 für große Teile der Öffentlichkeit desavouierte Franz Josef Strauß, der nun wieder ministrabel war. Dies führte dazu, dass Conrad Ahlers, neben Rudolf Augstein prominentes Opfer der „Spiegel-Affäre“, als Regierungssprecher in dieselbe Regierung eintrat, in der Strauß, seinerzeit der Hauptverantwortliche für den Coup gegen den „Spiegel“, Finanzminister wurde. Zudem saßen ehemalige NSDAP-Mitglieder (neben Kurt Georg Kiesinger auch Karl Schiller, Lauritz Lauritzen und Gerhard Schröder), frühere Kommunisten und Sozialisten respektive Widerstandskämpfer (Herbert Wehner, Willy Brandt) und einer der vormals profiliertesten Adenauer-Kritiker (Gustav Heinemann) am 
Kabinettstisch. ${ }^{7}$ Solche Liasionen, für manche unverzeihliche Mesalliancen, waren für viele zunächst eine unerträgliche Vorstellung - und stellten so einen Reife- und Belastungstest nicht nur für die Regierungsparteien, sondern für die politische Kultur der Bundesrepublik dar. Denn erstmals amtierte eine Bundesregierung, in der die einstmals die Weimarer Republik prägenden ideologischen Gegensätze personell vereint waren. Die demokratische Kooperation dieser Kräfte war, insbesondere nach den Erfahrungen der Adenauer-Ära, heikel und für die Bundesregierung eine neue Situation.

Die jüngere Historiographie deutet dieses Personaltableau der Großen Koalition inzwischen als geschichtspolitische Versöhnung der Republik (Winkler 2004b: 241; Wolfrum 2006: 229), doch genau diese Melange wurde seinerzeit teilweise als Affront der politischen Moral begriffen. Beispielhaft kann dies an der Intervention von Günter Grass abgelesen werden, der Anfang Dezember 1966 in zwei Offenen Briefen an Brandt und Kiesinger - veröffentlicht im sozialdemokratischen „Vorwärts“ - versuchte, die Große Koalition noch aufzuhalten. Grass hatte im Bundestagswahlkampf 1965 für die SPD ,getrommelt“ und attackierte nun das neue Regierungsbündnis als „miese Ehe“, an der die SPD ,zerbrechen“ und das ganze Land „unheilbaren Schaden“ nehmen könne. Die Jugend werde sich vom Staat abkehren und ,sich nach links und rechts verrennen". Vor allem die Verletzung politischer Moral kritisierend, schrieb er in seinem Brief an Kiesinger, „dass in diesem Land mit seiner immer noch nicht abgetragenen Hypothek, in diesem geteilten Land ohne Friedensvertrag das Amt des Bundeskanzlers niemals von einem Mann wahrgenommen werden darf, der schon einmal wider alle Vernunft handelte und dem Verbrechen diente, während andere daran zugrunde gingen, weil sie der Vernunft folgten und dem Verbrechen Widerstand boten“. In einem Artikel in der „Zeit“ zog er eine Parallele zu „1933“ und argumentierte, mit der Großen Koalition werde „der Nationalsozialismus wieder hoffähig gemacht“ und der Aufstieg der NPD weiter gefördert. Sein bündiges Fazit: „Unter den fragwürdigsten Umständen. Die SPD regiert“ (Grass 1969: 95-103). Während Kiesinger auf Grass' offenen Brief nicht reagierte, antwortete Brandt: Die Große Koalition sei „die be-

7 Ganz im Sinne des „pragmatischen Hinwegsehens über unterschiedliche Herkünfte“ (Schildt 2003: 47) unterschlug die offizielle Darstellung der Biografien der Regierungsmitglieder sämtliche Tätigkeiten in NS-Organisationen, siehe: Das Kabinett Kiesinger, in: Bulletin des Presse- und Informationsamtes der Bundesregierung, 07.12.1966, 12421247. Zur jüngeren biografischen Forschung und der jeweiligen Involvierung in den Nationalsozialismus vgl. exemplarisch Eibl 2001: 31-34; Oppelland 2002: 76-131; Lütjen 2007: 35-94, 218; Bahnsen 2008: 32-42; Hochstätter 2008: 24-47. 
grenzte, heute mögliche Alternative zum bisherigen Trott. [...] Wir werden in das neue Kapitel der deutschen Nachkriegsgeschichte wesentliche neue Elemente einführen. Dafür werden wir Verantwortung tragen und gerade das geistige Deutschland nicht enttäuschen“ (Brandt 1966: 96).

Auch Karl Jaspers, Sebastian Haffner und andere meldeten sich mit dramatischen Attacken zu Wort. Das politische Argumentieren mit Geschichte stand hier meist im Mittelpunkt. Doch jenseits der zwar möglichen, aber extrem knappen Mehrheit mit der FDP war für die SPD letztlich ein Argument für den Entschluss zur Großen Koalition entscheidend, das der kurz nach Bildung des Regierungsbündnisses verstorbene Fritz Erler so formulierte: „Die Bundesrepublik Deutschland gewinnt für das eigene Volk und für die Umwelt ein anderes Gesicht, wenn die CDU/CSU aufhören muss, Staatspartei zu sein und wenn die Sozialdemokraten als gleichberechtigter Partner mit einer starken Mannschaft in die Bundesregierung einziehen“(Erler 1968: 423).

\section{Fallbeispiele zur Geschichtspolitik der Großen Koalition}

Wenn für die Große Koalition insgesamt gelten mag, sie sei in der Wahrnehmung der Öffentlichkeit bis vor kurzem eine beinahe ,,vergessene Regierung“ gewesen (Schmoeckel/Kaiser 2005), so trifft dies erst recht für ihre Geschichtspolitik zu. Dies ist bemerkenswert, da der Rekurs auf Geschichtsbilder sowohl in der Entstehungsphase der neuen Regierung als auch während deren knapp dreijährigen Bestehens nicht zu übersehen ist. Bereits die Bildung der Koalition provozierte Kritik, die mit Geschichte argumentierte, nicht nur von SEDChef Walter Ulbricht, der das Bündnis umgehend in die Nähe der reaktionären Politik Hugenbergs von 1932 rückte. Herbert Wehner, der „Architekt“ der Koalition auf SPD-Seite, hielt dem entgegen, ,dass jeder, der den Versuch macht, diese Regierung in Beziehung zu Kräften und Absichten zu bringen, die unser Volk in das Unglück der zwölf Jahre gerissen haben, von Grund auf irrt" (Wehner 1966: 1241).

Hinzu kam, dass die Regierung Kiesinger-Brandt mehrere Themen auf der Agenda hatte, die unweigerlich historische Reminiszenzen und damit geschichtspolitische Deutungsfragen aufwarfen. Genannt seien die Auseinandersetzung um den Umgang mit dem Münchner Abkommen vom September 1938, die Kontroverse um die Verjährung von NS-Verbrechen, nicht zuletzt aber auch die Auseinandersetzungen um den Aufstieg der NPD, um die Notstandsgesetze und um die geplante Wahlrechtsänderung, die stets das Gespenst von Weimar in die politische Diskussion beförderten (Ullrich 2009). Zur Ver- 
deutlichung großkoalitionärer Deutungspolitik hier nur zwei Beispiele: Franz Josef Strauß, Finanzminister der Großen Koalition und CSU-Vorsitzender, bezeichnete das geplante Abkommen zur Nichtweiterverbreitung von Atomwaffen, das die BRD unterzeichnen sollte, als ein ,neues Versailles von kosmischen Ausmaßen“. Alt-Bundeskanzler Adenauer sah in diesem Vertrag einen „Morgenthau-Plan im Quadrat“ (zit. nach Radkau 1983: 325; Wolfrum 2005: 301). Beispiel zwei: Der Vorsitzende der SPD-Fraktion im Bundestag, Helmut Schmidt, sprach mit Blick auf die Wirtschaftspolitik der Vorgängerregierung Erhard von einer „Deflationspolitik à la Brüning“ (Schmidt 1968: 14).

\section{a) „Markstein in der Geschichte": Kiesingers Regierungserklärung}

Am 1. Dezember 1966 wählte der Deutsche Bundestag Kurt Georg Kiesinger mit großer Mehrheit zum dritten Bundeskanzler. Kiesinger erhielt jedoch nur 340 von 473 abgegebenen Stimmen, 109 Abgeordnete votierten gegen ihn, 23 enthielten sich der Stimme, eine war ungültig und 23 hatten nicht an der Wahl teilgenommen; neben der oppositionellen FDP zeigten damit mehrere Dutzend SPD-Parlamentarier die Ablehnung dieses Bündnisses. Knapp zwei Wochen später, am 13. Dezember, trug Kiesinger die Regierungserklärung der nun konstituierten Großen Koalition vor. Diese Gattung politischer Texte, gleichsam der „Modellfall politischer Kommunikation“ (Gottschlich 1989), ist - besonders in Koalitionsregierungen - stets das Ergebnis detaillierter Absprachen mit den einzelnen Ressorts (Korte 2002). Deshalb darf die von Kiesinger vorgetragene Erklärung als Regierungsprogramm des gesamten Kabinetts und aller beteiligten Parteien gelesen werden (Stüwe 2005: 73 f., 272 ff.). Im Folgenden geht es jedoch nicht um eine genaue Rekonstruktion der Rede und ihres Kontextes, sondern darum, ihren geschichtspolitischen Status zu erkunden: In welcher Weise wird Geschichte als Teil des Begründungsdiskurses für die Politik der Großen Koalition benutzt, welche Geschichtsbilder werden für die hier programmatisch entworfene Reformpolitik mobilisiert?

Die Rede hebt an mit der Rechtfertigung der neuen Regierung. Kiesinger bezieht sich schon im ersten Satz auf die der Bildung der Großen Koalition vorausgehende „lange schwelende Krise“ der Vorgängerregierung Erhard (Kiesinger 1966: 203). In den zurückliegenden Koalitionsverhandlungen habe sich die ,wohl bisher gründlichste Bestandsaufnahme der Möglichkeiten und Notwendigkeiten deutscher Politik“ (203) ergeben. Dass sich die Unionsparteien und die SPD erstmals zur gemeinsamen Regierungsbildung entschlossen, wertete er als einen „Markstein in der Geschichte der Bundesrepublik“ (203). 
Gleichwohl konzedierte er: „Die Sorgen vieler gelten den möglichen Gefahren einer Großen Koalition, der nur eine verhältnismäßig kleine Opposition gegenübersteht.“ (204) Solch verbreitete Bedenken gegenüber seiner Regierung, die Kiesinger in der Wendung „Missbrauch der Macht“ zusammenfasst, veranlassten ihn zu einer zweiten in die nahe Zukunft gerichteten Feststellung: Die Koalitionspartner wollten die gemeinsame Regierung nur auf Zeit, nur bis zum Ende der ohnehin verkürzten Legislaturperiode fortführen (204). Dabei ist der Blick in die Zukunft das gleichsam jeder Regierungserklärung am Beginn einer neuen Legislaturperiode aufgegebene Soll - sie muss Ziele darlegen. Und dies war für Kiesinger ein von ökonomischen Fragen dominiertes Programm. So widmete er weit mehr als die Hälfte seiner Rede der Wirtschaftspolitik, denn die Situation der Staatsfinanzen bot sich ihm als ein ,düsteres Bild' (204) dar. Kiesinger analysierte die Finanzpolitik der letzten Jahre und konstruierte gewissermaßen zwischen den Zeilen eine wirtschaftspolitische Versagensgeschichte der Vorgängerregierung Erhard, die die von ihm geführte Große Koalition unmittelbar legitimierte. Natürlich kritisierte er keine Personen direkt, aber er sprach von einem zuvor „eingeschlagenen Weg ins Defizit“ (205).

Im Hintergrund freilich drängte eine andere Zeitebene, jene Vergangenheit, die Kiesinger nur einmal direkt ansprach. Im Rahmen der Aufzählung der Politikfelder, die von Einsparungen oder gar Kürzungen betroffen sein sollten, erwähnte er auch die „Abwicklung von Kriegs- und Nachkriegsfolgen“. Diese Gesetzgebung, so Kiesinger, „sollte abgeschlossen werden“. Denn „wichtige Aufgaben der Zukunftsvorsorge (würden) sträflich vernachlässigt, wenn die kommenden Jahre durch neue Zahlungen für die Vergangenheit belastet würden“ (207). Die Verbrechen des Nationalsozialismus heißen hier , die Vergangenheit", Geschichte bleibt weitgehend unkonkret.

In den außenpolitischen Passagen seiner Rede streift er etwa auch kurz das Verhältnis zu Polen und sagt: „In weiten Schichten des deutschen Volkes besteht der lebhafte Wunsch nach einer Aussöhnung mit Polen, dessen leidvolle Geschichte wir nicht vergessen haben [...]" (214). Auch hier ist ,leidvolle Geschichte' der Platzhalter zwar nicht nur, aber besonders für die deutsche, für die nationalsozialistische Unterjochung Polens seit dem September 1939. Mit Blick auf die Tschechoslowakei unterstreicht der Bundeskanzler ebenfalls den Verständigungswillen und räumt ein, ,dass das unter Androhung von Gewalt zustande gekommene Münchner Abkommen von 1938 nicht mehr gültig ist“". Allerdings gebe es noch Probleme mit dem Staatsangehörigkeitsrecht. Die „sudetendeutschen Landsleute“ (215) und die anderen Vertriebenen und Flüchtlinge „haben, wie das tschechoslowakische Volk zuvor, bitteres Leid und Un- 
recht erfahren“ (215). Diese Zeit nennt er ein „trübe(s) Kapitel der Geschichte unserer Völker“ (215), das die Bundesregierung beenden wolle, um ,ein Verhältnis vertrauensvoller Nachbarschaft zu schaffen“ (215). „Bitteres Leid und Unrecht" - diese harten moralischen Vokabeln tauchen da auf, wo die Schuld auf zwei Schultern verteilt werden kann, aber die deutsche Schuld nicht einmal beim Namen genannt wird. Wo es um deutsches Leid geht, findet er starke Worte, so bezeichnet er die deutsche Teilung als ,geschichtswidrige und unnatürliche Zerreißung unseres Volkes“ (217). Für Israel, mit dem die Bundesrepublik im Jahr zuvor unter eher peinlichen denn würdigen Umständen diplomatische Beziehungen aufgenommen hatte, hat er vier Sätze übrig: „Unter Missbrauch des Namens unseres Volkes sind gegen jüdische Menschen grauenhafte Verbrechen begangen worden. Diese gestalteten unser Verhältnis zu Israel problematisch und schwierig. Es wurde durch die Aufnahme diplomatischer Beziehungen verbessert und gefördert. Die Bundesregierung wird auf diesem Wege weiter fortschreiten“" (219).

Kanzler Kiesinger spricht mehrere historische Ereignisse direkt an: die „Wirtschaftskrise 1932“" (206), die ,beiden Weltkriege“ (206), die Aufnahme diplomatischer Beziehungen mit der Sowjetunion 1955, an der Kiesinger seinerzeit als Delegationsmitglied teilgenommen hatte (214), das Münchner Abkommen von 1938 (215), den deutsch-französischen Vertrag von 1963 (217), den Marschallplan (219) sowie seine eigene letzte Rede im Bundestag im Jahre 1958 (214). In allgemeiner Sicht wird Geschichte unterschiedlich aufgerufen: „Deutschland war jahrhundertelang die Brücke zwischen West- und Osteuropa.“ (214). Oder: „Diese Regierung wird keine der großen Hilfen vergessen, die uns die Vereinigten Staaten in den vergangenen beiden Jahrzehnten geleistet haben" (215).

Doch stets dann, wenn es um Konflikte geht, bleibt Kiesinger nebulös oder spart das Thema ganz aus. So spricht er in der außenpolitischen Passage zu den USA zwar von deren „Sorgen und Problemen“ (215), aber die Reizvokabel „Vietnam-Krieg“ fällt nicht. Auch die bundesdeutsche Gegenwart bleibt jenseits der von ihm detailliert beschriebenen wirtschaftlichen Probleme diffus und wird nicht recht beim Namen genannt. Beispiel NPD: Am 7. und 20. November war der rechtsextremen Partei der Einzug in die Landesparlamente von Hessen und Bayern gelungen, wodurch im ganzen Land eine öffentliche Debatte über den Umgang mit der neuen Partei am rechten Rand entbrannte. In Kiesingers Regierungserklärung findet sich dazu nur eine indirekte Reaktion, indem er ganz am Ende seiner Ansprache die Besorgnis von Teilen der Öffentlichkeit in Sachen Große Koalition mit der Diskussion um die NPD verknüpft 
und ausruft: „Dies ist nicht die Geburtsstunde eines neuen Nationalismus in Deutschland, nicht in dieser Regierung, nicht in diesem Hohen Hause und nicht in unserem Volk! Auch bei den letzten Landtagswahlen [in Hessen und Bayern, H.S.] hat sich die überwältigende Mehrheit der Wähler [...] zu den demokratischen Parteien bekannt" (219). Freilich erwähnt er die NPD nicht. Auch die DDR ist für ihn weiterhin nur der „andere Teil Deutschlands“ (218). Damit korrespondierend, bleibt der Nationalsozialismus begriffslos. An der noch deutlichsten Stelle heißt es: „Die Bundesregierung verurteilt die Politik Hitlers, die auf die Zerstörung des tschechoslowakischen Staatsverbandes gerichtet war." (215).

Jenseits der genannten knappen Bemerkungen zur Judenverfolgung und der Okkupation der Tschechoslowakei findet sich in Kiesingers Rede keinerlei moralische Qualifizierung oder historische Konkretisierung der Jahre zwischen 1933 und 1945; keiner der Begriffe wie etwa ,Nationalsozialismus', ,Drittes Reich' oder ,NS-Regime' fällt - einen deutschen Staat als verantwortliches und handelndes Subjekt scheint es damals nicht gegeben zu haben. Als Platzhalter für diese Epoche fungiert die Formulierung der „letzte Krieg“, die er mehrfach benutzt $(215,218,219)$.

Der christdemokratische Bundeskanzler beschwört in seiner Regierungserklärung Geschichte zwar vereinzelt, jedoch immer dann, wenn damit manifeste Probleme verbunden sind, verschwindet diese Geschichte ins Ungreifbare, oft Unbenannte. Kiesingers weitgehendes Beschweigen der nationalsozialistischen Herrschaft erscheint einerseits noch geprägt von der korrespondierenden Mehrheitsmentalität der fünfziger Jahre, andererseits war seine Rede auch ein markanter Ausdruck einer Geschichtspolitik durch Ausklammern der „Vergangenheit". Jenseits der hier nicht zu erörternden Frage, welche Möglichkeiten einem vormaligen „Gelegenheitsnazi“ (Görtemaker 2004: 443) wie Kiesinger in solch einer Situation realistischerweise zur Verfügung standen, ist festzuhalten, dass die gesamte Regierungserklärung der Großen Koalition von punktuellen Geschichtsrekursen durchzogen, aber von der historischen Perspektive respektive Argumentation nicht dominiert ist, im Gegenteil: Geschichte scheint zwar unerlässlich zur Politikbegründung, aber angesichts der vielen, gleichsam an allen Ecken lauernden historischen Störpotenziale verbleibt sie im rhetorisch Allgemeinen - und war so von sekundärer Bedeutung. Die Geschichtsbezüge sind im quantitativen Ausmaß auffallend randständig, in der qualifizierenden Deutung bemerkenswert, enthaltsam'. Die Dominanz des auf die Gegenwart konzentrierten Krisenbewusstseins mag dafür mitbestimmend gewesen sein - ebenso wie der besondere Charakter der Großen Koalition. Je- 
denfalls hatten die Rahmenbedingungen einen historischen, von Konkretisierung weitgehend absehenden Vermeidungsdiskurs zur Folge. Conrad Ahlers bescheinigte Kiesinger später, dieser habe in der Großen Koalition als ein „wandelnder Vermittlungsausschuss“" agiert (zit. nach Görtemaker 2004: 446). Erkennt man darin ein besonderes diplomatisches Talent, so hat es sich bereits in seiner geschichtspolitisch höchst defensiven Regierungserklärung niedergeschlagen.

\section{b) „Heute lässt uns die Geschichte als Lehrmeisterin im Stich“. Die Ost- und Deutschlandpolitik am Beispiel des Umgangs mit dem Gedenktag 17. Juni}

„Wir wollen entkrampfen und nicht verhärten, Gräben überwinden und nicht vertiefen" (Kiesinger 1966: 218). Mit diesem programmatischen Schlüsselsatz aus Kiesingers Regierungserklärung war das Motto für die Entspannungspolitik gegenüber Osteuropa und der DDR geprägt, die sich die Große Koalition auf die Fahnen geschrieben hatte. US-Präsident Lyndon B. Johnson hatte zwei Monate zuvor das Politikziel für den Westen so formuliert: „Unsere Aufgabe ist es eine Aussöhnung mit dem Osten zu erreichen“" (zit. nach Görtemaker 2004: 462). Diesen Weg wollte die Große Koalition nun gehen, denn die vormalige „passive Politik der Konzentration auf Rechtsstandpunkte“ (Braunmühl 1973: 136) war praktisch gescheitert, wenngleich bereits Erhards Außenminister Gerhard Schröder mit seiner „Politik der Bewegung“ teilweise versucht hatte, von alten ideologischen Bindungen vorsichtig Distanz zu gewinnen (Eibl 2001: 257 ff., 415 ff.; Potthoff 1999: 43 ff.). Die Problemlage war komplex und höchst sensitiv: Es ging um das Angebot einer Gewaltverzichtserklärung, um eine Aussöhnung mit Polen und um die Anerkennung der Staatsgrenzen Polens, und es ging um die Frage der Gültigkeit des Münchner Abkommens von 1938. Ganz besonders ging es um die Gratwanderung im Umgang mit der DDR: einerseits den westdeutschen Alleinvertretungsanspruch nicht aufzugeben und die DDR als selbständigen Staat nicht anzuerkennen, andererseits trotzdem Erleichterungen im deutsch-deutschen Verhältnis zu erreichen. ${ }^{8}$

Freilich war die Ostpolitik der Großen Koalition hinsichtlich ihres Veränderungspotenzials und ihrer Ergebnisse schon im Februar 1967 in eine nahezu aussichtslose Situation geraten, als die Staaten des Warschauer Pakts in Reak-

8 Zur jüngeren Forschung vgl. etwa Vogtmeier 1996; Potthoff 1999; Schmidt 2001; Taschler 2001. 
tion auf die übereilte Aufnahme diplomatischer Beziehungen zwischen Rumänien und der Bundesrepublik die „Ulbricht-Doktrin“ beschlossen, wonach unter anderem ohne Anerkennung der DDR keine Normalisierung des Verhältnisses zur Bundesrepublik möglich war (Bender 2008: 139 f.; Potthoff 1999: 55 ff.). Endgültig in der Sackgasse befand sie sich dann durch die außenpolitischen Erschütterungen vor und nach der Niederschlagung des Prager Frühlings. Allerdings war die Eintrübung der Gesprächsatmosphäre zwischen Bonn und Moskau auch durch die unentschiedene Haltung der Großen Koalition in Sachen Atomwaffensperrvertrag ebenso wie durch das Erstarken der NPD bedingt; die Sowjetführung hatte nach den Wahlerfolgen der rechtsextremen Partei mit ihrem Interventionsrecht gemäß der Feindstaatenklausel der UN-Charta gedroht. Insgesamt wuchs so die Einsicht, zunächst in der SPD, dass ohne Berücksichtigung der UdSSR als der entscheidenden Machtzentrale keine Veränderung zu erreichen war.

Trotzdem ist erkennbar, dass man in Bonn ernsthaft bemüht war, Kiesingers Motto ,Entkrampfen statt Verhärten' Taten folgen zu lassen. Flexibilität war das Stichwort der Stunde, innen- wie außenpolitisch. Nicht nur die erstmals bekundete Bereitschaft zu direkten Regierungsverhandlungen mit der DDR ist hier zu nennen, sondern auch ein aus heutiger Sicht schier lächerlicher, aber damals symbolisch bedeutsamer Schritt: der Umstand, dass offizielle Schreiben der DDR an die Adresse der Bonner Regierung nun tatsächlich beantwortet wurden, hatte man doch bislang unter dem dogmatischen Einfluss der „Hallstein-Doktrin" konsequent jeden Brief ignoriert. So kam es zu einem Schriftwechsel zwischen dem Vorsitzenden des DDR-Ministerrates Willi Stoph und Kanzler Kiesinger.

Ein aufschlussreiches geschichtspolitisches Beispiel für den neuen Entkrampfungskurs ist der Umgang mit dem Gedenktag des 17. Juni (Wolfrum 1999: 249 ff.; Krämer 1996: 210 ff.). Dieses Datum war seit 1953, als es kurz nach dem Aufstand in der DDR zum „Tag der deutschen Einheit“", dem Nationalfeiertag der Bundesrepublik erkoren wurde, ein doppeltes Symbol: Zum einen sollten die alljährlich praktizierten Gedenkakte mit den unzähligen Gedenkreden landauf, landab den westdeutschen Willen zur Wiedervereinigung signalisieren und die gesamtdeutsche nationale Identität wachhalten. Zum anderen fungierte dieses Datum mit seinen öffentlichen Attacken auf die „Zone“ und das „Pankower Regime“ als polarisierendes Instrument des Kalten Krieges. So lag es nahe, just an diesem symbolisch bedeutsamen Punkt eine sichtbare Veränderung vorzunehmen, eine geschichtspolitische Chance zur Beglaubigung des neuen ost- und deutschlandpolitischen Kurses. 
Der erste Schritt war am 17. Juni 1967 zu besichtigen, als Kanzler Kiesinger die Gedenkrede beim alljährlichen Staatsakt im Deutschen Bundestag hielt (Kiesinger 1967). Gleich eingangs seiner Ansprache kritisierte er den „,bequemen Formelkult“ im Kontext des 17. Juni, „,der das tönende Wort an die Stelle mühevollen politischen Denkens und Handelns setzt. Und keine Gelegenheit verführt die dafür Anfälligen zu dieser Torheit eher als die jährliche Wiederkehr dieses Gedenktages.“ Kiesinger fügte hinzu: „Ich habe nicht die Absicht, bei dieser zur Tradition gewordenen Gedenkstunde im Haus des Deutschen Bundestages in diesen Fehler zu verfallen." Vielmehr wolle er „ohne Scheuklappen sehen, was ist, auch das, was in den vergangenen 14 Jahren geworden ist". Die Große Koalition habe sich ,zu einer neuen, beweglicheren Politik gegenüber dem Osten entschlossen“ (541). Diese „Politik der Entspannung“ könne aber nur eingebettet sein in den Prozess der Überwindung des Ost-WestKonflikts in Europa, denn, so die bemerkenswerte Deutung: „Deutschland, ein wiedervereinigtes Deutschland, hat eine kritische Größenordnung. Es ist zu groß, um in der Balance der Kräfte keine Rolle zu spielen, und zu klein, um die Kräfte um sich herum selbst im Gleichgewicht zu halten“ (542). Den „Verantwortlichen im anderen Teil Deutschlands", wie Kiesingers Formulierung lautete, bot er „Gespräche und Vereinbarungen“ zur Linderung von Spaltung und Not sowie zur Verbesserung der Beziehungen an: „Diese innere Entkrampfung oder Entgiftung entspräche unserem großen Entwurf einer künftigen europäischen Friedensordnung" (542). Dass er in dieser Rede darauf verzichtete, argumentative Hilfe aus der Historie zu suchen, begründete er am Ende seiner Ansprache so: „Wir alle - im Osten wie im Westen - stehen vor einer weltpolitischen Situation, für die es kein Vorbild, keine Präzedenzfälle gibt. Wenn je das Wort ,historia vitae magistra' einmal gelten konnte - heute lässt uns die Geschichte als Lehrmeisterin im Stich; ja es könnte sein, dass sie uns gar den Blick auf die Zukunft verstellt, die ganz neue Gedanken, neue Entwürfe, neuen unbefangenen Wagemut von allen Beteiligten fordert" (543).

Das waren für diesen deutschland- und geschichtspolitisch so wichtigen Jahrestag neue Töne. Schon zuvor hatte man erkannt, dass die bisherige Praxis des 17. Juni kontraproduktiv zu werden drohte. Im Februar 1967 verständigten sich CDU und SPD darauf, eine Kabinettsvorlage zur Streichung des 17. Juni als Feiertag auszuarbeiten. Dieser Tag, so die Begründung, passe nicht mehr zu einer ostpolitischen Friedenspolitik und dürfe nicht mehr als Waffe gegen sie missbraucht werden. In den Fraktionen der Regierungsparteien stieß das Vorhaben freilich auf massive Vorbehalte und geriet auf die lange Bank, denn der Diskussionsbedarf war erheblich. So brachte zunächst die oppositionelle FDP 
am 22. Juni 1967 einen Gesetzesvorschlag zur Aussetzung des Feiertagscharakters des 17. Juni bis zur Wiedervereinigung in den Bundestag ein, der freilich nach der Überweisung in den Ausschuss für gesamtdeutsche und Berliner Fragen nicht zur Abstimmung im Plenum gestellt wurde. Im Frühjahr 1968 legte dann die Große Koalition einen eigenen Gesetzentwurf vor, mit dem der 17. Juni als staatlicher und arbeitsfreier Feiertag gestrichen werden sollte, aber als „Nationaler Gedenktag des deutschen Volkes“, wie er seit der Proklamation von Bundespräsident Heinrich Lübke im Jahre 1963 offiziell hieß, erhalten bleiben sollte. Begründet wurde dieser Vorstoß nun mit dem Argument, die Bevölkerung habe in den letzten Jahren nach ,neuen Ausdrucksformen gesucht, um die innere Verbundenheit zwischen den beiden getrennten Teilen des deutschen Volkes zum Bewusstsein zu bringen“. Dabei sei der Status des 17. Juni als gesetzlicher Feiertag ,vielfach ein Hindernis und reihte ihn vor allem dort, wo die Voraussetzungen zur politischen Würdigung fehlten, zu leicht in die Reihe gewohnter arbeitsfreier Tage ein“ (zit. nach Krämer 1996: 214). Doch obwohl der Bundesrat bereits Zustimmung signalisiert hatte, erfolgte keine Gesetzesänderung, da die Koalitionsvorlage im Bundestagsausschuss auf scharfe Ablehnung stieß. Auch aus den Gewerkschaften gab es starke Vorbehalte, diesen Feiertag zu streichen, allerdings schien eine Kompensation durch gleichzeitige Einführung eines Bildungsurlaubes möglich. Doch letztlich war der Widerstand in den Unionsparteien zu stark, so dass die Große Koalition dieses Projekt nicht zu Ende führte.

Ausdruck dieser parteiübergreifenden Unsicherheit und Kritik an der bisherigen Praxis des 17. Juni war dann jener erinnerungskulturell-symbolpolitische Einschnitt des Jahres 1968, als erstmals kein Staatsakt, keine Gedenkstunde im Bundestag stattfand. Zwar gab es weiterhin im ganzen Land Gedenkfeiern, auf denen auch einzelne Regierungsmitglieder sprachen, auch der Bundeskanzler wandte sich in einer Radioansprache an die Bevölkerung, doch die Tradition war damit gebrochen. Im Jahr darauf, 1969, ging die Große Koalition auf diesem Weg noch einen Schritt weiter: Den jährlichen „Bericht zur Lage der Nation im geteilten Deutschland“, den alle Bundestagsfraktionen 1967 von der Bundesregierung forderten und diese erstmals im März 1968 vorlegte, verschob die Große Koalition nun auf den deutschlandpolitisch zentralen Jahrestag. Regierungsbericht und parlamentarische Aussprache statt Gedenken - so lautete die neue Bonner Tagesordnung des 17. Juni, die eher der Einsicht in die Vorteile sachlich-diskursiver Formen entsprungen war denn veränderter deutschlandpolitischer Überzeugungen. 
Kiesinger erstattete also am 17. Juni 1969 dem Bundestag den Bericht und verband dies mit einem Gedenken an den 20. Jahrestag der Gründung der Bundesrepublik (Kiesinger 1969b). Zwar hatte sich nun der Ton der Rede Kiesingers deutlich verschärft, was vor allem an der koalitionszerrüttenden Wahl Gustav Heinemanns zum Bundespräsidenten im März 1969 lag, gleichfalls an dem bereits laufenden Bundestagswahlkampf sowie besonders an der DDRFührung, die den Initiativen der Großen Koalition nicht nur die kalte Schulter gezeigt, sondern Maßnahmen zur weiteren Abschottung getroffen hatte. Trotzdem blieb die innenpolitisch mobilisierende und in der Auseinandersetzung mit der SED anklagende Funktion des 17. Juni Vergangenheit. Kiesinger sprach nun sogar mehrfach ausdrücklich von der DDR - allerdings noch apostrophiert. Dass im selben Jahr etwa auch ein Buch mit dem sprechenden Titel „Die DDR ist keine Zone mehr" erschien (Schwarze 1969), illustriert diese bis in die Regierungsspitze hineinreichende gesellschaftliche Wahrnehmungsveränderung.

Doch entgegen seiner oben zitierten Auffassung des Jahres 1967 benutzte Kiesinger in dieser Rede Geschichte wieder als politisches Argument. So erinnerte er im Zusammenhang der „Studentenunruhen“ an den „Untergang der Weimarer Republik“: Wo immer sich Tendenzen zeigten, „die sich grundsätzlich gegen unsere freiheitliche gesellschaftliche und staatliche Ordnung richten, müssen wir schon den Anfängen wehren“. Die Weimarer Republik sei „nicht nur an der Not der Massenarbeitslosigkeit, sondern auch an der geistigen und politischen Zerrissenheit des deutschen Volkes zugrunde gegangen“. Der Kanzler erkannte Parallelen zwischen der gewalttätigen und „,zunehmenden Radikalisierung des politischen Lebens" am Ende Weimars und der in der Bundesrepublik „,seit den Ostertagen des vergangenen Jahres [...] wieder offen und herausfordernd“ auftretenden Gewalttätigkeit. Gelänge es nicht, „für Ordnung in unserem eigenen Hause zu sorgen“, bestünde die „Gefahr, dass die Gewalttaten der linksextremen Gruppen überhandnehmen und zugleich einen von diesen Gewalttätigkeiten aufgeschreckten Teil unserer Bevölkerung in den Rechtsextremismus treiben" (Kiesinger 1969b: 673 f.). Dieser Ausflug Kiesingers in die Geschichte ist in zweierlei Hinsicht bemerkenswert: Nicht nur, dass er bei der Ursachenanalyse des Endes der Weimarer Demokratie strikt jede Aussage vermeidet, welche politischen Kräfte für deren Exitus hauptverantwortlich waren, sondern auch der Umstand, dass er in der Analyse gegenwärtiger politischer Gewalt ungenau ist und von der Ursache der „Osterunruhen“ 1968, dem Mordanschlag auf Rudi Dutschke, schweigt. Indirekt entsteht so bei ihm die These, Rechtsextremismus sei eine Folge von Linksextremismus. 
Die Große Koalition hatte in ihrer Ost- und Deutschlandpolitik mit der Degradierung und Umfunktionierung des 17. Juni zwar geschichtspolitisch neue Signale gesetzt (von denen die Unionsparteien freilich in ihrer Oppositionszeit seit Ende 1969 nichts mehr wissen wollten), auch die im Juli 1968 veröffentlichte Erklärung der Bundesregierung zur Ungültigkeit des Münchner Abkommens zählt hierzu. Insgesamt gelangen ihr wichtige Schritte in der Anpassung an die veränderte weltpolitische Lage, nicht zuletzt die informelle Annullierung der Hallstein-Doktrin. Jedoch scheiterte sie mit ihrem Ziel einer Entspannung auch der Beziehungen zur DDR; dazu genügte die reformpolitisch zunächst nur symbolisch bedeutsame Rücknahme des Status des Gedenktages 17. Juni nicht. Dies konnte, wie wir heute wissen, nur durch den Umweg über Moskau gelingen, da der Widerstand der SED-Führung mit entgegenkommender Geschichtsund Symbolpolitik alleine nicht zu überwinden war. Eine innovative Geschichtspolitik kann auf solchen Reformwegen wichtige Beglaubigungsschritte liefern, muss freilich Teil einer insgesamt stimmigen und glaubwürdigen Politik sein. Dies gelang erst der sozial-liberalen Koalition unter Willy Brandt.

\section{c) „Kein Ermächtigungsgesetz": Die Notstandsgesetze}

In seiner ursprünglichen Fassung enthielt das Grundgesetz keine Bestimmung, mit der auf die besonderen Bedingungen eines Angriffs von außen oder einer Gefährdung der inneren Sicherheit hätte reagiert werden können. Die Frage wurde aktuell mit der Erlangung weitgehender Souveränität im Jahre 1955. Grundlage hierfür war der 1952 mit den Westmächten abgeschlossene Deutschlandvertrag. Ein Passus des Vertragswerks garantierte den Westmächten unter bestimmten Bedingungen Vorbehaltsrechte für die Erklärung eines Notstandes in der Bundesrepublik. Seit 1957 hatte die mit absoluter Mehrheit wiedergewählte Regierung Adenauer versucht, diese staatsrechtliche Lücke der bundesdeutschen Verfassungsstruktur zu korrigieren. Damit begann eine über zehnjährige Kontroverse, die zu den herausragenden politischen Auseinandersetzungen der jungen Bundesrepublik zählt und von parlamentarischen Debatten und einer breiten außerparlamentarischen Protestbewegung gekennzeichnet war (Schneider 1986; Krohn 1981). In den jahrelangen Debatten standen sich zwei Positionen gegenüber: „Ging es der einen Seite um die Verabschiedung einer gesetzlich genau definierten Vorsorge für den Notstandsfall, die den demokratischen Rechtsstaat auch in Extremsituationen vor dem Zusammenbruch bewahren sollte, sah die andere Seite in jeder Notstandsgesetzgebung 
bereits eine generelle Ermächtigung der Exekutive zur Beseitigung der Demokratie" (Schönhoven 2004: 268).

Nachdem in den Jahren 1960, 1962 und 1965 drei Versuche gescheitert waren, im Bundestag eine entsprechende Verfassungsänderung zu verabschieden, verabredeten Union und SPD, während der Zeit der Großen Koalition das Problem endlich zu lösen. Der Kompromiss, auf den sich die Regierung schließlich einigte, sah für den Notstandsfall die Errichtung eines „Gemeinsamen Ausschusses“ aus Mitgliedern des Bundestages und des Bundesrates vor, der als eine Art Notparlament die parlamentarischen Rechte aufrechterhalten sollte. Zudem gelang es der SPD, diverse Kritikpunkte aufzunehmen, besonders hervorzuheben ist die Verankerung eines Widerstandsrechts in Art. 20 des Grundgesetzes.

Die sich formierende außerparlamentarische Protestbewegung mündete in das 1966 gegründete „Kuratorium Notstand der Demokratie“. Im Mai 1968 demonstrierten etwa 150.000 Menschen gegen die Verabschiedung der Gesetze. Die Anti-Notstandsbewegung war dabei ein wesentlicher Motor auch einer geschichtspolitischen Zuspitzung der breiten gesellschaftlichen Debatte (Spernol 2008). Doch trotz beträchtlicher Mobilisierungserfolge gelang es nicht, die Grundgesetzänderung zu verhindern. Mitentscheidend für die letztlich gescheiterte Protestbewegung war der Umstand, dass die Gewerkschaften, die den Kampf gegen die Notstandspläne seit 1958 angeführt hatten, sich in der Schlussphase von dem Protestbündnis mit der sich stetig radikalisierenden APO lösten und einen Generalstreik ablehnten. Nach langen Verhandlungen, in denen diverse Monita der Kritiker aufgenommen wurden, beschloss der Bundestag am 30. Mai 1968 die dazu nötigen Grundgesetzänderungen für eine Notstandsverfassung.

Welche historischen Argumente spielten dabei eine Rolle? Bereits gegen den ersten Gesetzentwurf, den der damalige CDU-Innenminister Gerhard Schröder 1960 vorlegte, hagelte es Proteste, vor allem seitens der Gewerkschaften, die darin einen „massiven Angriff auf unsere Staatsordnung“ erkannten. Sie fühlten sich an den berüchtigten Notverordnungsparagraphen 48 der Weimarer Reichverfassung (WRV) erinnert, manche meinten gar, der Gesetzentwurf Schröders sei „schlimmer als die entsprechenden Nazigesetze“ (zit. nach Schneider 1986: 57 f., 64). Während der Debatte um die Verabschiedung im Bundestag in den Jahren 1967 und 1968 tobte ein Deutungskampf um die Frage, ob Bonn doch Weimar sei; linksliberale Intellektuelle, Schriftsteller und Wissenschaftler jedenfalls und die ihnen nahestehende Publizistik und vor allem das gesamte gewerkschaftliche Milieu waren in Alarmstimmung. Der im 
Frühjahr 1967 verzeichnete, sprunghafte Anstieg auf knapp 700.000 Erwerbslose und der scheinbar ungebremste Aufstieg der NPD, die am 28. April 1968 in Baden-Württemberg mit fast zehn Prozent der Stimmen bereits in den siebten Landtag einzog, nährten immer wieder Krisenszenarien, die Vergleiche mit „Weimarer Verhältnissen“ hervorriefen. Dabei bewirkte die Verquickung der Debatte mit aufsehenerregenden aktuellen Ereignissen, die scheinbar Befürchtungen ob eines bevorstehenden Polizeistaates nährten, noch eine Verstärkung der ohnehin schon dramatisierenden Deutungen. So etwa, als der berüchtigte Besuch des Schah von Persien zu den bekannten öffentlichen Protesten führte, in deren Zusammenhang der Student Benno Ohnesorg von einem Polizist erschossen wurde, und nur wenige Wochen später die erste parlamentarische Beratung des Notstandsgesetzeswerks stattfand.

In den Protestkundgebungen gegen das Vorhaben der Großen Koalition wurden die Notstandsgesetze polemisch als „NS-Gesetze“ abgekürzt - damit schien alles gesagt (Spernol 2008). Das Vorhaben als solches galt als Ausdruck und Instrument eines „neuen Faschismus“. So wurden auf Demonstrationen Transparente gezeigt, auf denen es hieß: „1933 Ermächtigungsgesetz - 1968 Notstandsgesetz" (Coverfoto Schneider 1986). Flugblätter verbreiteten die Losung „Einmal 1933 ist genug“ (zit. nach Schneider 1986: 244, 250) oder „Wehrt Euch jetzt... ehe es wieder zu spät ist!“ (Titelzeile der Streikzeitung des „Kuratoriums Notstand der Demokratie“, Mai 1968, http://www.dhm.de). Ernst Bloch etwa, der am 30. Oktober 1966 die Abschlussrede vor über 20.000 Teilnehmern des Frankfurter Kongresses „Notstand der Demokratie“ hielt, sah die Zeit gekommen, „den Anfängen zu wehren“ und erkannte in dem „Notstandsunrecht" eine ,warnende Ähnlichkeit mit der gehabten Weimarer Demokratie und ihrem diktatorischen Ende" (Bloch 1980).

Solche und ähnliche historisch angereicherten Argumente bestimmten die außerparlamentarische Debatte, so dass die Große Koalition in diesem Fall zu einer defensiven Geschichtspolitik gedrängt war. Hauptargument war, es gehe darum, einen machtpolitischen Notstandsmissbrauch zu verhindern, wie in der Weimarer Republik mit dem Art. 48 der WRV geschehen. „Es ist nicht wahr, dass dieser Entwurf den Weg zur Diktatur bereitet", so der CDU-Abgeordnete Carl Otto Lenz am 15. Mai 1968 im Bundestag (zit. nach Schneider 1986: 251). Angesichts der geschichtspolitisch aufgeladenen außerparlamentarischen Proteste war Innenminister Paul Lücke erkennbar darauf bedacht, historische Begründungen für den Eingriff in das Grundgesetz auf ein Minimum zu beschränken. Einerseits setzte er sich mit den Motiven auseinander, weshalb der Parlamentarische Rat 1948/49 keine Notstandsbestimmungen ins Grundgesetz 
aufgenommen hatte. Andererseits versuchte er, die gewissenhafte Beachtung rechtsstaatlicher Standards im Regierungsentwurf nachzuweisen. Auf Kritik, die Notstandsregelung sei zu ausführlich, antwortete er: „Die kürzeste Regelung wäre eine Generalklausel. Jedoch gerade hier schrecken die Spuren der jüngeren deutschen Vergangenheit. Und nicht zuletzt das Modell einer Notstandsnorm, die die Machthaber jenseits der Mauer in ihrem Verteidigungsgesetz [...] dekretiert haben". Kontrastierend hob der CDU-Innenminister die rechtsstaatlichen Eckpunkte der Notstandsgesetze hervor und resümierte: „Im Hinblick auf dieses vielfältige System der Sicherungen widerlegt sich die Behauptung von selbst, die Notstandsverfassung stelle ein Ermächtigungsgesetz zur legalen Einführung der Diktatur dar" (Lücke 1967a: 591). Kanzler Kiesinger verstärkte dieses Argument noch und sagte bei der abschließenden Beratung im Bundestag: „Nicht eine politische oder militärische Diktatur, sondern ihre Verhinderung auch für den Fall der äußeren Gefahr ist doch das Ziel dieser Gesetze!“(Kiesinger 1969c: 196)

Stellten sich Regierungsmitglieder jedoch den Kritikern außerhalb des Parlaments, konnten sie den geschichtspolitischen Argumenten nicht ausweichen. So wehrte sich Gerhard Jahn (SPD), Parlamentarischer Staatsekretär im Auswärtigen Amt, in einer öffentlichen Diskussion gegen die Vergleiche mit dem Ende der Weimarer Republik. Er entgegnete: „Das Jahr 1933 war nicht durch institutionelle Sicherungen zu verhindern. Eine so vollständige Änderung der politischen Lage ist auf diese Weise nicht zu vermeiden. Das ist vielmehr eine Frage des Verhaltens der Demokraten in solchen Situationen. [...] In der Diskussion um die Gefahr eines Missbrauchs der Notstandsverfassung belasten die historischen Erfahrungen die Diskussion. Es ist ein größeres Maß an Vertrauen zur Demokratie in der Bundesrepublik und zu unserer demokratischen Verfassungsstruktur notwendig, um auf diese Weise die Diskussion in eine etwas weniger durch historische Analogien belastete Atmosphäre zu führen.“ Und weiter sagte Jahn: „Wenn man die Ereignisse der deutschen Geschichte, auf die hier angespielt worden ist, nicht wieder haben will, dann ist es unsere Aufgabe, in der gegenwärtigen Lage demokratisches Bewusstsein zu zeigen. Man kann sich nicht bei der Diskussion um gegenwärtige Fragen ständig nur auf die historischen Erfahrungen berufen". Gegen den Vorwurf, unhistorisch und bloß formal zu argumentieren, setzte Jahn die Erwiderung, „,dass derjenige, der ständig die Geschichte bemüht, nur nach rückwärts gewandt ist“". Beriefe man sich immer nur auf schlechte historische Erfahrungen, werde man nie dazu kommen, bessere Regelungen zu finden (Jahn 1967: 118, 121, 124). 
Das hochsuggestive politisch-historische Symbol „Notstand“ dominierte die Auseinandersetzungen um diese Grundgesetzänderung. Geschichtspolitisch konnte die Große Koalition den so plakativen wie emotionalisierenden Angriffen der Gegner einer Notstandsverfassung wenig Paroli bieten, drangen doch ihre nüchternen und sachlichen Rechtfertigungen in der erhitzten Debatte kaum durch. Die politische Emotionalisierung gründete in meist alles andere als willkürlichen, freilich schlagkräftig miteinander verknüpften Deutungen: in der auf die Weimar- und NS-Erfahrung rekurrierenden Diktaturangst, in der dramatisch als demokratiegefährdend beurteilten Notstandsgesetzgebung der Regierung sowie in dem biografisch-politischen Misstrauen gegenüber einem Teil der Koalition. Dagegen ließ sich geschichtspolitisch nur defensiv argumentieren, auch deshalb, weil das Notstandsthema narrativ in erster Linie Bedrohungsszenarien weckte und es ermöglichte, die zeitweise verbreitete demokratiepolitische Sorge mit Personal und Vorhaben der Regierung zu identifizieren. Durchaus anders sah die Lage im Falle der Kontroverse um ein neues Wahlrecht aus.

\section{d) „Bonn ist noch nicht Weimar": Die Wahlrechtsreform}

In der Regierungserklärung Kiesingers fungierte das politische Ziel, eine Wahlrechtsreform zu beschließen, als Argument zur Beschwichtigung der Kritiker des Regierungsbündnisses, denn mit dem angepeilten Mehrheitswahlrecht sollte ein ,institutioneller Zwang zur Beendigung der Großen Koalition und eine institutionelle Abwehr der Notwendigkeit von Koalitionen überhaupt geschaffen" werden (Kiesinger 1966: 204). Die Lösung dieser Frage, so Kiesinger ein Jahr später, sei die „eigentliche Bewährungsprobe“ der Großen Koalition (zit. nach Morsey 2000: 108), allerdings, und darin war er sich mit Willy Brandt einig, halte er diese Frage im Unterschied zu Innenminister Lücke nicht für die "schlechthin entscheidende nationale Frage“ (zit. nach Schönhoven 2004: 257, 266). Gerade dieses Reformwerk allerdings wurde nicht verabschiedet.

Der federführende Innenminister Lücke setzte eine Expertenkommission aus Politologen und Juristen ein (Mitglieder waren u.a. Thomas Ellwein, Theodor Eschenburg und Wilhelm Hennis), die im Dezember 1967 mit großer Mehrheit für die sofortige Einführung eines relativen Mehrheitswahlrechts plädierte. Wenngleich sich die Regierungsparteien anfangs in diesem Ziel einig waren, erwies sich der innerparteiliche Widerstand in der SPD letztlich als zu groß. Was für die CDU zunächst ein Instrument gegen die ungeliebte FDP war, 
und zwischenzeitlich auch mit Blick auf die Wahlerfolge der NPD gerechtfertigt wurde, verwandelte sich in den Augen der Sozialdemokraten zusehends in eine Beschränkung der eigenen Machtchancen. Als der Nürnberger Parteitag der SPD im März 1968 beschloss, das Projekt für zwei Jahre auf Eis zu legen, trat Innenminister Lücke zurück. Nach der Wahl Heinemanns zum Bundespräsidenten mit den Stimmen der FDP im März 1969 war allen Beteiligten klar, dass sich die SPD nicht mehr auf eine Wahlrechtsreform einlassen würde, die sie um den möglicherweise erforderlichen Partner für eine sozialdemokratisch geführte Regierung bringen würde (vgl. Schönhoven 2004: 233 ff.).

Die geschichtspolitische Begründung einer Wahlrechtsreform hatte vor allem einen Fluchtpunkt - Weimar. Und bemerkenswerterweise war es in dieser Diskussion besonders jener Innenminister Lücke, der sich in der Notstandsdebatte so sehr mit historischen Argumenten zurückgehalten hatte, aber in der Wahlrechtsauseinandersetzung immer wieder mit Geschichtsbildern hantierte. „Die deutsche Demokratie will die negativen Erfahrungen aus dieser Zeit [der Weimarer Republik, H.S.] nutzen. Die Deutschen wollen nicht, dass ihre parlamentarische Demokratie wieder an der eigenen Schwäche, an parteipolitischer Zersplitterung zugrunde geht. Sie wollen nicht, dass Rechts- und Linksradikalismus sich unter Missbrauch der freiheitlichen Grundordnung daranmacht, diese Demokratie zu zerstören." Zur Veranschaulichung seiner Position argumentierte er kontrafaktisch: „Wie wäre wohl die deutsche und die Weltgeschichte verlaufen, wenn Deutschland etwa 1924/26 das für die parlamentarische Demokratie so wichtige Mehrheitswahlrecht eingeführt hätte?" (Lücke 1967b). Lücke gab zwar keine Antwort darauf, zeigte sich aber gewiss, dass ein Mehrheitswahlrecht ,das entscheidende Mittel [ist], unsere Demokratie auf Dauer zu stabilisieren. Weimar ist uns allen noch in Erinnerung. [...] Die politischen Fehler der Vergangenheit sollten und müssen für uns Wegweiser für die Zukunft sein“ (Lücke 1967c: 431). Den geschichtspolitischen Vorwurf an die Adresse der Großen Koalition, sie begebe sich mit einer solchen Wahlrechtsänderung ,in die Nähe der Ermächtigungsgesetze von Hitler aus dem Jahre 1933“, konterte Lücke gleichfalls mit einem historischen Argument: „Die Weimarer Regierung wurde verächtlich gemacht. Auch das war einer der Gründe für ihren Niedergang“" (Lücke 1967c: 431). Wie schon erwähnt, trat Lücke nach dem Scheitern der Wahlrechtsreform von seinem Ministeramt zurück. Kurz darauf veröffentlichte er ein Buch mit dem Titel „Ist Bonn doch Weimar?“ Zwar räumte er darin ein: „Bonn ist noch nicht Weimar.“ Doch die jüngsten „Alarmzeichen der Geschichte“ seien genug Warnung, endlich ein Mehrheitswahlrecht einzuführen (Lücke 1968: 16, 90). 
Anders als im Falle der Notstandskontroverse setzte die Große Koalition in der Begründung der Wahlrechtsreform auch auf historische Argumente. Neben dem Expertenkriterium der sachlichen Angemessenheit zeigt sich dabei das Moment der politischen Opportunität: Politik braucht Geschichte - aber nur, wenn sie auch nützlich ist; werden Geschichtsbilder von politischen Kontrahenten gegen die eigenen Ziele mobilisiert, ohne selbst entsprechend kontern zu können, geraten politische Akteure schnell in die Defensive und begründen ihre Vorhaben ahistorisch.

\section{Geschichte als Problem - ein Fazit}

Als das Regierungsbündnis von Unionsparteien und Sozialdemokratie im Herbst 1966 gebildet wurde, gab es nicht wenige Warnungen mit dem historischen Verweis auf die Erfahrungen von Weimar. Knapp drei Jahre später schienen diese Bedenken entkräftet. Im Sommer 1969, als sich die Große Koalition bereits ihrem Ende zuneigte, waren publizistische Entwarnungssignale zu lesen: „Weimar hatte Überdruck, Bonn leidet an Unterdruck; die Agonie der ersten Republik war wie ein hitziges Fieber, der Zustand der zweiten ähnelt mehr einer Schlafkrankheit“, schrieb der Journalist Hans Schuster. „Man darf sich daher nicht durch die Angstvorstellung irritieren lassen, dass Bonn unweigerlich Weimar immer ähnlicher werde. Sofern sie nicht einem Zweckpessimismus dient, verrät sie eine Zwangsvorstellung: wer sich ihr hingibt, bildet sich ein Leiden ein, während er die tatsächlichen Schwächen unserer heutigen Gesellschaft übersieht" (Schuster 1969: 513).

Angesichts des explorativen Charakters dieser Studie können hier nur einige begrenzte Schlussfolgerungen gezogen werden. Die Analyse der in den Fallstudien zur Regierungserklärung, zum 17. Juni, zu den Notstandsgesetzen und zur Wahlrechtsreform herangezogenen Quellen kann zwar nicht beanspruchen, die gesamte Geschichtspolitik der Großen Koalition zu erfassen, hat allerdings Hinweise auf Problemlagen und Verhaltensweisen erbracht, die weiteren vertiefenden Studien ersten Aufschluss über die geschichtspolitische Gemengelage der Handelnden geben können.

In dieser Hinsicht befand sich die Große Koalition über weite Strecken in einer defensiven Position. Einerseits war die CDU/CSU/SPD-Regierung teilweise konfrontiert mit massiver Kritik ihrer politischen Gegner aus dem gesellschaftlichen Umfeld, die mittels plakativer, pejorativer und hochemotionalisierender Geschichtsbilder Reformprojekte der Regierung torpedierten. Andererseits fehlten ihr ähnlich attraktive, affirmative historische Gegenbilder. 
So wählte sie eher den Weg der Vermeidung, mithin des opportunistischen Umgangs mit Geschichte. Dies war zunächst in einer spezifischen politischen Konstellation begründet: dem Zusammentreffen grundlegender Reformvorhaben mit einer breiten gesellschaftlichen Aufbruchsbewegung. Doch auch der alle Politikfelder bestimmende Kompromisscharakter der Regierung Kiesinger/Brandt war hierfür entscheidend.

Hinzu kam, dass die knapp drei Regierungsjahre bis 1969 als Zeit technokratischer Politik zu verstehen sind, einem stark auf Zukunft ausgerichteten Planungsdenken (Metzler 2005), dem Geschichte oftmals eine bestenfalls sekundäre Größe schien. So war Geschichte als Orientierungs- und Begründungselement von Politik für die Große Koalition eher ein Problem als ein lohnenswerter Fundus. Gegen die Dominanz negativer Geschichtsbilder verblassten die Politik- und Zukunftsentwürfe teilweise, so dass temporale Legitimität ein so schwieriges wie knappes Gut war. Auch aus diesem Grund war sie bestrebt, auf wichtigen Politikfeldern Distanz zur Geschichte zu bekommen. Wo historische Bezüge nicht zu umgehen waren, hatte sie einen schweren Stand. Da die Orientierungsinstanz ,Geschichte' gleichzeitig in der Gesellschaft eine regelrechte Konjunktur erlebte, befand sich die Politikbegründung der Großen Koalition in einer widersprüchlichen Situation. Nicht nur die Vielzahl von Projekten, Ereignissen und Personen, die in den Jahren 1966 bis 1969 nahezu zwangsläufig bedrohliche historische Assoziationen evozierten, auch die noch geringe zeitliche Nähe zu Hitler und Weimar standen einer offensiven Geschichtspolitik zur Legitimation der eigenen Reformvorhaben entgegen. Insofern kann der Zusammenhang von ,Reform und Geschichte' für die Regierungszeit der Großen Koalition partiell als Aporie verstanden werden.

\section{Literatur}

Allemann, Fritz René, 1956: Bonn ist nicht Weimar, Köln u. a.

Bahnsen, Uwe, 2008: Karl Schiller, Hamburg.

Bahr, Egon, 1998: Zu meiner Zeit, München.

Baring, Arnulf, 1982: Machtwechsel. Die Ära Brandt-Scheel, Stuttgart.

Bender, Peter, 2008: Deutschlands Wiederkehr. Eine ungeteilte Nachkriegsgeschichte 1945-1990, Bonn.

Bloch, Ernst, 1980: [Rede auf dem Kongreß Notstand der Demokratie], in: Jürgen Miermeister/Jochen Staadt (Hrsg.), Provokationen. Die Studenten- 
und Jugendrevolte in ihren Flugblättern 1965-1971, Darmstadt u. a., 150152.

Böll, Heinrich, 1969: Vorwort, in: Beate Klarsfeld, Kiesinger. Die Geschichte des PG 2633 930. Dokumentation mit einem Vorwort von Heinrich Böll, Darmstadt, 7-8.

Brandt, Willy, 1966: [Schreiben an Günter Grass], in: Günter Grass, 1969: Über das Selbstverständliche. Politische Schriften, München (neu durchgesehene und ergänzte Ausgabe), 96-97.

Brandt, Willy, 1992: Erinnerungen. Mit einem aktuellen Vorwort, Frankfurt am Main/Berlin.

Braunmühl, Claudia von, 1973: Kalter Krieg und friedliche Koexistenz. Die Außenpolitik der SPD in der Großen Koalition, Frankfurt am Main.

Buchstab, Günter (Bearb.), 2005: Kiesinger: „Wir leben in einer veränderten Welt". Die Protokolle des CDU-Bundesvorstands 1965-1969, Düsseldorf.

Buchstab, Günter/Gassert, Philipp/Lang, Peter Thaddäus (Hrsg.), 2005: Kurt Georg Kiesinger 1904-1988. Von Ebingen ins Kanzleramt, Freiburg u. a.

Dittberner, Jürgen, 2006: Große Koalition - kleine Schritte. Politische Kultur in Deutschland, Berlin.

Dittberner, Jürgen, 2007: Große Koalition: 1966 und 2005, in: Aus Politik und Zeitgeschichte, B 35-36, 11-18.

Ehmke, Horst, 1980: [Debattenbeitrag] in: Deutscher Bundestag (Hrsg.), Zur Verjährung nationalsozialistischer Verbrechen. Dokumentation der parlamentarischen Bewältigung des Problems 1960-1979, Teil II, Bonn, 381390.

Eibl, Franz, 2001: Politik der Bewegung. Gerhard Schröder als Außenminister 1961-1966, München.

Eichhorn, Joachim Samuel, 2006: „Mehr als einige schöne Trinksprüche“. Die Konsensstrategien der ersten Großen Koalition (1966-1969), in: Zeithistorische Forschungen/Studies in Contemporary History 3 (2), 231-246.

Eichhorn, Joachim Samuel, 2009: Durch alle Klippen hindurch zum Erfolg. Die Regierungspraxis der ersten Großen Koalition (1966-1969), München.

Erler, Fritz, 1968: Zur Großen Koalition, in: Ders., Politik für Deutschland. Eine Dokumentation. Mit einem Vorwort von Willy Brandt, hrsg. und eingeleitet von Wolfgang Gaebler, Stuttgart (2. Aufl.), 422-424.

Fabian, Christine/Rössel, Uta (Bearb.), 2009: Die Kabinettsprotokolle der Bundesregierung. Bd. 19: 1966, hrsg. von Hartmut Weber, München. 
Frese, Matthias/Paulus, Julia/Teppe, Karl (Hrsg.), 2005: Demokratisierung und gesellschaftlicher Aufbruch. Die sechziger Jahre als Wendezeit der Bundesrepublik, Paderborn u. a. (2. Aufl.).

Fröhlich, Claudia/Heinrich, Horst-Alfred (Hrsg.), 2004: Geschichtspolitik. Wer sind ihre Akteure, wer ihre Rezipienten?, Stuttgart.

Gassert, Philipp, 2006: Kurt Georg Kiesinger 1904-1988. Kanzler zwischen den Zeiten, München.

Gassert, Philipp, 2008: Zweimal Große Koalitionen: 1966 bis 1969 und seit 2005, in: Hans-Peter Schwarz (Hrsg.), Die Bundesrepublik Deutschland. Eine Bilanz nach 60 Jahren, München, 99-119.

Görtemaker, Manfred, 2004: Geschichte der Bundesrepublik Deutschland. Von der Gründung bis zur Gegenwart, Frankfurt am Main.

Gottschlich, Maximilian, 1989: Regierungserklärungen als Modellfälle politischer Kommunikation, in: Ders./Oswald Panagl/Manfried Welan (Hrsg.), Was die Kanzler sagten. Regierungserklärungen der Zweiten Republik 1945-1987, Wien u. a., 33-68.

Grass, Günter, 1969: Über das Selbstverständliche. Politische Schriften, München (neu durchgesehene und ergänzte Ausgabe).

Greve, Michael, 2001: Der justizielle und rechtspolitische Umgang mit den NS-Gewaltverbrechen in den sechziger Jahren, Frankfurt am Main.

Hall, Peter A., 1993: Policy Paradigms, Social Learning, and the State. The Case of Economic Policymaking in Britain, in: Comparative Politics 25 (3), 275-296.

Hildebrand, Klaus, 1984: Von Erhard zur Großen Koalition: 1963-1969, Stuttgart.

Hildebrand, Klaus, 2006: Die erste Große Koalition 1966 bis 1969. Gefährdung oder Bewährung der parlamentarischen Demokratie in der Bundesrepublik?, in: Zeitschrift für Parlamentsfragen 37 (3), 611-625.

Hochstätter, Matthias, 2008: Karl Schiller. Eine wirtschaftspolitische Biografie, Saarbrücken.

Hofmann, Werner/Maus, Heinz (Hrsg.), 1967: Notstandsordnung und Gesellschaft in der Bundesrepublik Deutschland. Zehn Vorträge, Reinbek.

Jahn, Gerhard, 1967: Diskussionsbeiträge, in: Werner Hofmann/Heinz Maus (Hrsg.), Notstandsordnung, 117-124.

Kiesinger, Kurt Georg, 1966: Regierungserklärung vor dem Deutschen Bundestag am 13. Dezember 1966, in: ders., 1969a: Stationen 1949-1969, Tübingen, 203-220. 
Kiesinger, Kurt Georg, 1967: Mit Ernst und Redlichkeit der Freiheit und Einheit unseres Volkes dienen, in: Bulletin des Presse- und Informationsamtes der Bundesregierung, 20. Juni, 541-543.

Kiesinger, Kurt Georg, 1969a: Stationen 1949-1969, Tübingen.

Kiesinger, Kurt Georg, 1969b: Verantwortung für die Einheit der Nation und des Vaterlandes, in: Bulletin des Presse- und Informationsamtes der Bundesregierung, 18. Juni, 669-674.

Kiesinger, Kurt Georg, 1969c: Erklärung vor dem Deutschen Bundestag zur Verabschiedung der Notstandsgesetze, in: Ders., Die Große Koalition 19661969. Reden und Erklärungen des Bundeskanzlers, hrsg. von Dieter Oberndörfer, Stuttgart 1979, 195-199.

Klöckler, Jürgen, 2005: Kurt Georg Kiesinger im „Dritten Reich“. Der nachmalige baden-württembergische Ministerpräsident als frühes NSDAPParteimitglied und der Holocaust, in: Zeitschrift für Württembergische Landesgeschichte 64, 355-364.

Knorr, Heribert, 1974: Die Große Koalition in der parlamentarischen Diskussion der Bundesrepublik von 1949 bis 1965, in: Aus Politik und Zeitgeschichte, B 33, 24-47.

Knorr, Heribert, 1975: Der parlamentarische Entscheidungsprozess während der Großen Koalition 1966 bis 1969. Struktur und Einfluss der Koalitionsfraktionen und ihr Verhältnis zur Regierung der Großen Koalition, Meisenheim am Glan.

Kohlstruck, Michael, 2004: Erinnerungspolitik: Kollektive Identität, Neue Ordnung, Diskurshegemonie, in: Birgit Schwelling (Hrsg.), Politikwissenschaft als Kulturwissenschaft. Theorien, Methoden, Problemstellungen, Wiesbaden, 173-193.

König, Helmut, 2008: Politik und Gedächtnis, Weilerswist.

Korte, Karl-Rudolf(Hrsg.), 2002: „Das Wort hat der Herr Bundeskanzler“. Eine Analyse der Großen Regierungserklärungen von Adenauer bis Schröder, Wiesbaden.

Krämer, Martin, 1996: Der Volksaufstand vom 17. Juni 1953 und sein politisches Echo in der Bundesrepublik Deutschland, Bochum.

Kroegel, Dirk, 1997: Einen Anfang finden! Kurt Georg Kiesinger in der Außenund Deutschlandpolitik der Großen Koalition, München.

Krohn, Maren, 1981: Die gesellschaftlichen Auseinandersetzungen um die Notstandsgesetze, Köln.

Longerich, Peter, 1995: Deutschland 1918-1933. Die Weimarer Republik, Hannover. 
Lücke, Paul, 1967a: Unser Staat muss auch in der Stunde der Not ein Rechtsstaat bleiben, in: Bulletin des Presse- und Informationsamtes der Bundesregierung, 30. Juni, 591-595.

Lücke, Paul, 1967b: Lebensfähige Demokratie durch politische Bildung, in: Bulletin des Presse- und Informationsamtes der Bundesregierung, 17. Februar, 129.

Lücke, Paul, 1967c: Die Bedeutung des Wahlrechts für die Demokratie, in: Bulletin des Presse- und Informationsamtes der Bundesregierung, 12. Mai, 430-432.

Lücke, Paul, 1968: Ist Bonn doch Weimar? Der Kampf um das Mehrheitswahlrecht, Frankfurt am Main u. a.

Lütjen, Torben, 2007: Karl Schiller (1911-1994). „Superminister“ Willy Brandts, Bonn.

März, Peter, 2007: Große Koalitionen. Deutsche und weitere Erfahrungen, in: Einsichten und Perspektiven. Bayerische Zeitschrift für Politik und Geschichte, Themenheft 1/2007, 3-63.

Marx, Stefan (Bearb.), 2010: Die CDU/CSU-Fraktion im Deutschen Bundestag, Sitzungsprotokolle 1966-1969, Düsseldorf.

Metzler, Gabriele, 2005: Konzeptionen politischen Handelns von Adenauer bis Brandt. Politische Planung in der pluralistischen Gesellschaft, Paderborn u. a.

Metzler, Gabriele, 2005: Die Reformprojekte der Großen Koalition im Kontext ihrer Zeit, in: Günter Buchstab/Philipp Gassert/Peter Thaddäus Lang (Hrsg.), Kurt Georg Kiesinger 1904-1988. Von Ebingen ins Kanzleramt, Freiburg u. a., 421-452.

Morsey, Rudolf, 2000: Die Bundesrepublik Deutschland, München (4., überarb. und erw. Aufl.).

Morsey, Rudolf, 2005: Die Große Koalition - Vorgeschichte und Nachwirkungen, in: Günter Buchstab/Philipp Gassert/Peter Thaddäus Lang (Hrsg.), Kurt Georg Kiesinger 1904-1988, Freiburg u.a., 393-419.

Niclauß, Karlheinz, 2008: Kiesinger und Merkel in der Großen Koalition, in: Aus Politik und Zeitgeschichte, B 16, 3-10.

Oppelland, Torsten, 2002: Gerhard Schröder (1910-1989). Politik zwischen Staat, Partei und Konfession, Düsseldorf.

Picht, Georg, 1964: Die deutsche Bildungskatastrophe. Analyse und Dokumentation, Olten u. a.

Potthoff, Heinrich, 1999: Im Schatten der Mauer. Deutschlandpolitik 1961 bis 1990, Berlin. 
Prasse, Jan-Ole, 2010: Der kurze Höhenflug der NPD. Rechtsextreme Wahlerfolge der 1960er Jahre, Marburg.

Radkau, Joachim, 1983: Aufstieg und Krise der deutschen Atomwirtschaft 1945-1975. Verdrängte Alternativen in der Kerntechnik und der Ursprung der nuklearen Kontroverse, Reinbek.

Rohe, Karl, 1994: Politik. Begriffe und Wirklichkeiten. Eine Einführung in das politische Denken, Stuttgart u. a. (2., völlig überarb. u. erw. Aufl.).

Rohe, Karl, 1996: Politische Kultur: Zum Verständnis eines theoretischen Konzepts, in: Oskar Niedermayer/Klaus von Beyme (Hrsg.), Politische Kultur in Ost- und Westdeutschland, Opladen, 1-21.

Rosin, Philip, 2007: Kurt Georg Kiesinger und Charles de Gaulle. Die Entwicklung der deutsch-französischen Beziehungen in der Zeit der Großen Koalition 1966-1969, Bonn.

Schildt, Axel/Siegfried, Detlef/Lammers, Karl Christian (Hrsg.), 2003: Dynamische Zeiten. Die 60er Jahre in den beiden deutschen Gesellschaften, Hamburg (2. Aufl.).

Schildt, Axel, 2003: Materieller Wohlstand - pragmatische Politik - kulturelle Umbrüche. Die 60er Jahre in der Bundesrepublik, in: Axel Schildt/Detlef Siegfried/Karl Christian Lammers (Hrsg.), 2003: Dynamische Zeiten. Die 60er Jahre in den beiden deutschen Gesellschaften, Hamburg (2. Aufl.), 21-53.

Schildt, Axel, 2005: Nur die Farben gleichen sich. Zur Begründung der großen Koalition kann das erste schwarz-rote Bündnis nur bedingt taugen. $\mathrm{Zu}$ anders waren die Voraussetzungen, zu anders die handelnden Personen, Die Tageszeitung, 21. November, 11.

Schildt, Axel/Siegfried, Detlef, 2009: Deutsche Kulturgeschichte. Die Bundesrepublik 1945 bis heute, München.

Schmid, Harald, 2001: Erinnern an den „Tag der Schuld“. Das Novemberpogrom von 1938 in der deutschen Geschichtspolitik, Hamburg.

Schmid, Harald, 2007a: „Man kann wieder wählen!“ Aufstieg und Fall der NPD 1964-1969, in: Deutschland Archiv 40 (1), 122-130.

Schmid, Harald, 2008: Konstruktion, Bedeutung, Macht. Zum kulturwissenschaftlichen Profil einer Analyse von Geschichtspolitik, in: Horst-Alfred Heinrich/Michael Kohlstruck (Hrsg.), Geschichtspolitik und sozialwissenschaftliche Theorie, Stuttgart, 75-98.

Schmid, Harald, 2009: Vom publizistischen Kampfbegriff zum Forschungskonzept. Zur Historisierung der Kategorie „Geschichtspolitik“, in: Ders. 
(Hrsg.), Geschichtspolitik und kollektives Gedächtnis. Erinnerungskulturen in Theorie und Praxis, Göttingen, 53-75.

Schmid, Thomas, 2007: Wir Zufriedenen, Die Welt, 07.04.07, 1.

Schmidt, Helmut, 1968: Am Anfang war die Krise, in: SPD-Fraktion im Deutschen Bundestag (Hrsg.), 147 mal Soll und Haben. Zwischenergebnisse sozialdemokratischer Politik in der Großen Koalition, Bad Godesberg, 11-17. Schmidt, Wolfgang, 2001: Kalter Krieg, Koexistenz und kleine Schritte. Willy Brandt und die Deutschlandpolitik 1948-1963, Wiesbaden.

Schmoeckel, Reinhard/Kaiser, Bruno, 2005: Die vergessene Regierung. Die große Koalition 1966-1969 und ihre langfristigen Wirkungen, Bonn (2. Aufl.).

Schneider, Andrea H., 1999: Die Kunst des Kompromisses. Helmut Schmidt und die Große Koalition 1966-1969, Paderborn u. a.

Schneider, Franz, 1969: Große Koalition - Ende oder Neubeginn? München.

Schneider, Michael, 1986: Demokratie in Gefahr? Der Konflikt um die Notstandsgesetze: Sozialdemokratie, Gewerkschaften und intellektueller Protest (1958-1968), Bonn.

Schöllgen, Gregor, 2003: Willy Brandt. Die Biographie, München.

Schönhoven, Klaus, 2004: Wendejahre. Die Sozialdemokratie in der Zeit der Großen Koalition 1966-1969, Bonn.

Schroers, Rolf (Hrsg.), 1968: Der demokratische Obrigkeitsstaat. Beiträge zur demokratischen Funktionsfähigkeit der großen Koalition, Bonn.

Schuster, Hans, 1967: Die ,gerechte“ Koalition. Ideologie und Praxis des Bonner Bündnisses, in: Merkur 21, 801-815.

Schuster, Hans, 1969: Wird Bonn doch Weimar?, in: Merkur 23, 501-514.

Schwarze, Hanns Werner, 1969: Die DDR ist keine Zone mehr, Köln.

Spernol, Boris, 2008: Notstand der Demokratie. Der Protest gegen die Notstandsgesetze und die Frage der NS-Vergangenheit, Essen.

Steinbach, Peter, 2001: Geschichte und Politik - nicht nur ein wissenschaftliches Verhältnis, in: Aus Politik und Zeitgeschichte, B 28, 3-7.

Strauß, Franz Josef, 1991: Die Erinnerungen, Berlin.

Strohmeier, Gerd, 2009: Große Koalitionen in Deutschland und Österreich, in: Zeitschrift für Politikwissenschaft 19 (1), 5-37.

Stüwe, Klaus, 2005: Die Rede des Kanzlers. Regierungserklärungen von Adenauer bis Schröder, Wiesbaden.

Taschler, Daniela, 2001: Vor neuen Herausforderungen. Die außen- und deutschlandpolitische Debatte in der CDU/CSU-Bundestagsfraktion während der Großen Koalition (1966-1969), Düsseldorf. 
Tüffers, Bettina (Bearb.), 2009: Die SPD-Fraktion im Deutschen Bundestag. Sitzungsprotokolle 1966-1969, Düsseldorf.

Türk, Henning, 2006: Die Europapolitik der Großen Koalition 1966-1969, München

Ullrich, Sebastian, 2009: Der Weimar-Komplex. Das Scheitern der ersten deutschen Demokratie und die politische Kultur der frühen Bundesrepublik 1945-1959, Göttingen.

Vogtmeier, Andreas, 1996: Egon Bahr und die deutsche Frage. Zur Entwicklung der sozialdemokratischen Ost- und Deutschlandpolitik vom Kriegsende bis zur Vereinigung, Bonn.

Walser, Martin, 1986: Geschichte als Zeughaus, in: ders., Geständnis auf Raten, Frankfurt am Main, 84-86.

Weber, Petra, 1998: Carlo Schmid 1896-1979. Eine Biographie, Frankfurt am Main.

Wehner, Herbert, 1966: Verantwortung gegenüber dem ganzen deutschen Volk, in: Bulletin des Presse- und Informationsamtes der Bundesregierung, 03.12., 1241-1242.

Winkler, Heinrich August (Hrsg.), 2004a: Griff nach der Deutungsmacht. Zur Geschichte der Geschichtspolitik in Deutschland, Göttingen.

Winkler, Heinrich August, 2004b: Der lange Weg nach Westen II. Deutsche Geschichte 1933-1990, Bonn.

Wolfrum, Edgar, 1999: Geschichtspolitik in der Bundesrepublik Deutschland. Der Weg zur bundesrepublikanischen Erinnerung 1948-1990, Darmstadt. Wolfrum, Edgar, 2005: Die Bundesrepublik Deutschland 1949-1990, Stuttgart. Wolfrum, Edgar, 2006: Die geglückte Demokratie. Geschichte der Bundesrepublik Deutschland von ihren Anfängen bis zur Gegenwart, Stuttgart.

Korrespondenzanschrift:

Dr. Harald Schmid

Christian-Albrechts-Universität zu Kiel

Historisches Seminar

Olshausenstraße 40

24098 Kiel

E-Mail: haraldschmid@email.uni-kiel.de

Web: http://www.histosem.uni-kiel.de/Lehrstuehle/pohl/person/schmid.html 